This article appeared in "Singularities, Algebraic Geometry, Commutative Algebra and Related Topics. Festschrift for Antonio Campillo on the Occasion of his 65th Birthday." G.-M. Greuel, L. Narváez and S. Xambó-Descamps eds., pp. 55-106, Springer, 2018. https://doi.org/10.1007/978-3-319-96827-8_3

\title{
ULTRAMETRIC SPACES OF BRANCHES ON ARBORESCENT SINGULARITIES
}

\author{
EVELIA R. GARCÍA BARROSO, PEDRO D. GONZÁLEZ PÉREZ, AND PATRICK POPESCU-PAMPU
}

This paper is dedicated to Antonio Campillo and Arkadiusz Ptoski.

\begin{abstract}
Let $S$ be a normal complex analytic surface singularity. We say that $S$ is arborescent if the dual graph of any good resolution of it is a tree. Whenever $A, B$ are distinct branches on $S$, we denote by $A \cdot B$ their intersection number in the sense of Mumford. If $L$ is a fixed branch, we define $U_{L}(A, B)=(L \cdot A)(L \cdot B)(A \cdot B)^{-1}$ when $A \neq B$ and $U_{L}(A, A)=0$ otherwise. We generalize a theorem of Płoski concerning smooth germs of surfaces, by proving that whenever $S$ is arborescent, then $U_{L}$ is an ultrametric on the set of branches of $S$ different from $L$. We compute the maximum of $U_{L}$, which gives an analog of a theorem of Teissier. We show that $U_{L}$ encodes topological information about the structure of the embedded resolutions of any finite set of branches. This generalizes a theorem of Favre and Jonsson concerning the case when both $S$ and $L$ are smooth. We generalize also from smooth germs to arbitrary arborescent ones their valuative interpretation of the dual trees of the resolutions of $S$. Our proofs are based in an essential way on a determinantal identity of Eisenbud and Neumann.
\end{abstract}

\section{Contents}

1. Introduction

2. A reminder on intersection theory for normal surface singularities

2.1. The determinant of a normal surface singularity

2.2. Mumford's rational intersection number of branches

3. Generalities on ultrametrics and trees

3.1. Trees, rooted trees, arborescent partial orders and hierarchies

3.2. Ultrametric spaces and dated rooted trees

3.3. Additive distances on trees

4. Arborescent singularities and their ultrametric spaces of branches

4.1. Determinant products for arborescent singularities

4.2. The ultrametric associated to a branch on an arborescent singularity

4.3. Płoski's theorem and the ultrametric nature of Eggers-Wall trees

5. Valuative considerations

5.1. Basic types of valuations and semivaluations

5.2. The valuative partial order for arborescent singularities

6. Perspectives on non-arborescent singularities

6.1. Non-arborescent examples

6.2. Some open problems

References

2000 Mathematics Subject Classification. 14J17 (primary), 32S25.

Key words and phrases. Additive distance, Branch, Hierarchy, Mumford intersection number, Nef cone, Normal surface singularity, Resolution, Rooted tree, Ultrametric, Valuation. 


\section{INTRODUCTION}

Płoski proved the following theorem in his paper [42]:

"Let $f, g, h \in \mathbb{C}\{x, y\}$ be irreducible power series. Then in the sequence

$$
\frac{m_{0}(f, g)}{(\operatorname{ord} f)(\operatorname{ord} g)}, \frac{m_{0}(f, h)}{(\operatorname{ord} f)(\operatorname{ord} h)}, \frac{m_{0}(g, h)}{(\operatorname{ord} g)(\operatorname{ord} h)}
$$

there are two terms equal and the third is not less than the equal terms. Here $m_{0}(f, g)$ denotes the intersection multiplicity of the branches $f=0, g=0$ and ord $f$ stands for the order of $f$."

Denote by $Z_{f} \subset\left(\mathbb{C}^{2}, 0\right)$ the branch (that is, the germ of irreducible curve) defined by the equation $f=0$. One has analogously the branches $Z_{g}, Z_{h}$. Looking at the inverses of the previous quotients:

$$
U\left(Z_{f}, Z_{g}\right):=\left\{\begin{array}{cc}
\frac{(\operatorname{ord} f)(\operatorname{ord} g)}{m_{0}(f, g)}, & \text { if } Z_{f} \neq Z_{g} \\
0, & \text { if } Z_{f}=Z_{g}
\end{array},\right.
$$

one may express Płoski's theorem in the following equivalent way:

$U$ is an ultrametric distance on the set of branches of $\left(\mathbb{C}^{2}, 0\right)$.

Note that, if $Z_{f}$ and $Z_{g}$ are two different branches and if $l \in \mathbb{C}\{x, y\}$ defines a smooth branch transversal to $Z_{f}$ and $Z_{g}$ (that is, if one has ord $f=m_{0}(l, f)$ and ord $g=m_{0}(l, g)$ ), then:

$$
U\left(Z_{f}, Z_{g}\right)=\frac{m_{0}(l, f) m_{0}(l, g)}{m_{0}(f, g)}
$$

This is the view-point we take in our paper. Instead of working with multiplicities, we work with intersection multiplicities (also called intersection numbers) with a fixed branch. More precisely, we study the properties of the quotients:

$$
U_{L}(A, B):=\left\{\begin{array}{l}
\frac{(L \cdot A)(L \cdot B)}{A \cdot B}, \text { if } A \neq B, \\
0, \text { if } A=B,
\end{array}\right.
$$

when $A$ and $B$ vary in the set of branches of a normal surface singularity $S$ which are different from a fixed branch $L$. In the previous formula, $A \cdot B \in \mathbb{Q}_{+}^{*}$ denotes Mumford's intersection number of [36, II.b] (see Definition 17).

We focus on the germs of normal surfaces which have in common with $\left(\mathbb{C}^{2}, 0\right)$ the following crucial property: the dual graphs of their resolutions with simple normal crossings are trees. We call arborescent the normal surface singularities with this property. Note that in this definition we impose no conditions on the genera of the irreducible components of exceptional divisors (see also Remark 65).

We prove that (see Theorem 85):

Let $S$ be an arborescent singularity and let $L$ be a fixed branch on it. Then, $U_{L}$ is an ultrametric distance on the set of branches of $S$ different from $L$.

Given a finite set $\mathcal{F}$ of branches on $S$, one gets in this way an infinite family of ultrametric distances on it, parametrized by the branches $L$ which do not belong to $\mathcal{F}$. But, whenever one has an ultrametric on a finite set $\mathcal{F}$, there is a canonically associated rooted tree whose set of leaves is $\mathcal{F}$ (the interior-rooted tree of Definition 45). In our context, we show that the previous infinite family of ultrametrics define all the same unrooted tree. This tree may be interpreted in the following way using the resolutions of $S$ (see Theorem 87 and Remark 101):

Let $S$ be an arborescent singularity and let $\mathcal{F}$ be a finite set of branches on it. Let $L$ be another branch, which does not belong to $\mathcal{F}$. Then the interior-rooted tree associated to $U_{L}$ is homeomorphic to the union of the geodesics joining the representative points of the 
branches of $\mathcal{F}$ in the dual graph of any embedded resolution of the reduced Weil divisor whose branches are the elements of $\mathcal{F}$.

Both theorems are based on the following result (reformulated differently in Proposition 69):

Let $S$ be an arborescent singularity. Consider a resolution of it with simple normal crossing divisor $E$. Denote by $\left(E_{v}\right)_{v \in \mathcal{V}}$ the irreducible components of $E$ and by $\left(\check{E}_{v}\right)_{v \in \mathcal{V}}$ their duals, that is, the divisors supported by $E$ such that $\check{E}_{v} \cdot E_{w}=\delta_{v w}$, for any $v, w \in \mathcal{V}$ and $\delta_{v w}$ denotes the Kronecker delta. Then, for any $u, v, w \in \mathcal{V}$ such that $v$ belongs to the geodesic of the dual tree of $E$ which joins $u$ and $w$, one has:

$$
\left(-\check{E}_{u} \cdot \check{E}_{v}\right)\left(-\check{E}_{v} \cdot \check{E}_{w}\right)=\left(-\check{E}_{v} \cdot \check{E}_{v}\right)\left(-\check{E}_{u} \cdot \check{E}_{w}\right) \text {. }
$$

In turn, this last result is obtained from an identity between determinants of weighted trees proved by Eisenbud and Neumann [18, Lemma 20.2] (see Proposition[70 below). Therefore, our proof is completely different in spirit from Płoski's original proof, which used computations with Newton-Puiseux series. Instead, we work exclusively with the numbers $-\check{E}_{u} \cdot \check{E}_{v}$, which are positive and birationally invariant, in the sense that they are unchanged if one replaces $E_{u}$ and $E_{v}$ by their strict transforms on a higher resolution (see Corollary 20).

We were also inspired by an inequality of Teissier [45, Page 40]:

Let $S$ be a normal surface singularity with marked point $O$. If $A, B$ are two distinct branches on it and $m_{O}$ denotes the multiplicity function at $O$, then one has the inequality:

$$
\frac{m_{O}(A) \cdot m_{O}(B)}{A \cdot B} \leq m_{O}(S)
$$

We prove the following analog of it in the setting of arborescent singularities (see Corollary 84, in which we describe also the case of equality):

Whenever $L, A, B$ are three pairwise distinct branches on the arborescent singularity $S$ and $E_{l}$ is the unique component of the exceptional divisor of an embedded resolution of $L$ which intersects the strict transform of $L$, one has:

$$
U_{L}(A, B) \leq-\check{E}_{l} \cdot \check{E}_{l} .
$$

Then, in Theorem 92, we prove the following analog of the fact that $U_{L}$ is an ultrametric:

Under the hypothesis that the generic hyperplane section of an arborescent singularity $S$ is irreducible, the function $U_{O}$ defined by the left-hand side of Teissier's inequality is also an ultrametric.

Our approach allows us also to extend from smooth germs to arbitrary arborescent singularities $S$ a valuative intepretation given by Favre and Jonsson [20, Theorem 6.50] of the natural partial order on the rooted tree defined by $U_{L}$.

Namely, consider a branch $A$ on $S$ different from $L$, and an embedded resolution of $L+A$. As before, we denote by $\left(E_{v}\right)_{v \in \mathcal{V}}$ the irreducible components of its exceptional divisor. Look at the dual graph of the total transform of $L+A$ as a tree rooted at the strict transform of $L$, and denote by $\preceq_{L}$ the associated partial order on its set of vertices, identified with $\mathcal{V} \cup\{L, A\}$. To each $v \in \mathcal{V}$ is associated a valuation $\operatorname{ord}_{v}^{L}$ of the local ring $\mathcal{O}$ of $S$, proportional to the divisorial valuation of $E_{v}$ and normalized relative to $L$. Similarly, to the branch $A$ is associated a semivaluation $\operatorname{int}_{A}^{L}$ of $\mathcal{O}$, which is also normalized relative to $L$, defined by $\operatorname{int}_{A}^{L}(h)=\left(A \cdot Z_{h}\right) \cdot(A \cdot L)^{-1}$ (see Definition [116). Given two semivaluations $\nu_{1}$ and $\nu_{2}$, say that $\nu_{1} \leq$ val $\nu_{2}$ if $\nu_{1}(h) \leq \nu_{2}(h)$ for all $h \in \mathcal{O}$. This is obviously a partial order on the set of semivaluations of $\mathcal{O}$. We prove (see Theorem 119):

For an arborescent singularity, the inequality $\operatorname{ord}_{u}^{L} \leq{ }_{v a l} \operatorname{ord}_{v}^{L}$ is equivalent to the inequality $u \preceq_{L} v$. Similarly, the inequality $\operatorname{ord}_{u}^{L} \leq_{v a l} \operatorname{int}_{A}^{L}$ is equivalent to the inequality $u \preceq_{L} A$.

Even when $S$ is smooth, this result is stronger than the result of Favre and Jonsson, which concerns only the case where the branch $L$ is also smooth. In this last case, our Theorem 87 specializes to Lemma 
3.69 of [20], in which $U_{L}(A, B)$ is expressed in terms of what they call the relative skewness function $\alpha_{x}$ on a tree of conveniently normalized valuations (we give more explanations in Remark 118).

As was the case for Płoski's treatment in [42, Favre and Jonsson's study in [20] is based in an important way on Newton-Puiseux series. We avoid completely the use of such series and we extend their results to all arborescent singularities, by using instead the dual divisors $\check{E}_{u}$ defined above. Our treatment in terms of the divisors $\check{E}_{u}$ and the numbers $-\check{E}_{u} \cdot \check{E}_{v}$ was inspired by the alternative presentation of the theory of [20] given by Jonsson in [32, Section 7.3], again in the smooth surface case.

As in this last paper, our study could be continued by looking at the projective system of embedded resolutions of divisors of the form $L+C$, for varying reduced Weil divisors $C$, and by gluing accordingly the corresponding ultrametric spaces and rooted trees (see Remark 121). One would get at the limit a description of a quotient of the Berkovich space of the arborescent singularity $S$. We decided not to do this in this paper, in order to isolate what we believe are the most elementary ingredients of such a construction, which do not depend in any way on Berkovich theory.

Let us mention also another difference with the treatments of smooth germs $S$ in [20] and [32]. In both references, the authors treat simultaneously the relations between triples of functions on their trees (called skewness, thinness and multiplicity). Our paper shows that an important part of their theory (for instance, the reconstruction of the shape of the trees from valuation theory) may be done by looking at only one function (the one they call the skewness).

In the whole paper, we work for simplicity with complex normal surface singularities. Note that, in fact, our techniques make nearly everything work for singularities which are spectra of normal 2-dimensional local rings defined over algebraically closed fields of arbitrary characteristic. Indeed, our treatment is based on the fact that the intersection matrix of a resolution of the singularity is negative definite (see Theorem 3 below), a theorem which is true in this greater generality (see Lipman [35, Lemma 14.1]). The only exception to this possibility of extending our results to positive characteristic is Section 4.3], as it uses Newton-Puiseux series, which behave differently in positive characteristic (see Remark 114).

The paper is structured as follows. In Section 2 we recall standard facts about Mumford's intersection theory on normal surface singularities. In Section 3 we present basic relations between ultrametrics, arborescent posets, hierarchies on finite sets, trees, rooted trees, height and depth functions on rooted trees, and additive distances on unrooted ones. Even if those relations are standard, we could not find them formulated in a way adapted to our purposes. For this reason we present them carefully. The next two sections contain our results. Namely, the ultrametric spaces of branches of arborescent singularities are studied in Section 4 and the valuative interpretations are developed in Section 5 . We dedicate a special subsection (4.3) to the original case considered by Płoski, where both $S$ and $L$ are smooth, by giving an alternative proof of his theorem using so-called Eggers-Wall trees. Finally, Section [6 contains examples and a list of open problems which turn around the following question: is it possible to extend at least partially our results to some singularities which are not arborescent? Our examples show that there exist normal surface singularities and branches $L$ on them for which $U_{L}$ is not even a metric.

Since the first version of this paper, we have greatly extended its results, in collaboration with Matteo Ruggiero (see [25]), modifiying also substantially our approach: instead of working with rooted trees associated with ultrametrics, we work with unrooted trees associated with metrics satisfying the so-called four point condition. Nevertheless, we feel that this first approach remains interesting and potentially useful in other contexts.

\section{A REMINDER ON INTERSECTION THEORY FOR NORMAL SURFACE SINGULARITIES}

In this section we introduce the basic vocabulary and properties needed in the sequel about complex normal surface singularities $S$ and about the branches on them. In particular, we recall the notions of good resolution, associated dual graph, natural pair of dual lattices and intersection form on $S$. We explain the notion of determinant of $S$ and the way to define, following Mumford, a rational intersection number of effective divisors without common components on $S$. This definition is based in turn on the definition of the exceptional transform of such a divisor on any resolution of $S$. The exceptional transform belongs to the nef cone of the resolution. We show that the exceptional divisors belonging to the interior of the 
nef cone are proportional to exceptional transforms of principal divisors on $S$, a fact which we use later in the proof of Theorem 119 ,

\subsection{The determinant of a normal surface singularity.}

In the whole paper, $(S, O)$ denotes a complex analytic normal surface singularity, that is, a germ of complex analytic normal surface. The germ is allowed to be smooth, in which case it will still be called a singularity. This is a common abuse of language. Most of the time we will write simply $S$ instead of $(S, O)$.

Definition 1. A resolution of $S$ is a proper bimeromorphic morphism $\pi: \tilde{S} \rightarrow S$ with total space $\tilde{S}$ smooth. By abuse of language, we will also say in this case that $\tilde{S}$ is a resolution of $S$. The exceptional divisor $E:=\pi^{-1}(O)$ of the resolution is considered as a reduced curve. A resolution of $S$ is good if its exceptional divisor has simple normal crossings, that is, if all its components are smooth and its singularities are ordinary double points.

A special case of the so-called Zariski main theorem (see [30, Cor. 11.4]) implies that $E$ is connected, hence the associated weighted dual graph is also connected:

Definition 2. Let $\pi: \tilde{S} \rightarrow S$ be a good resolution of $S$. We denote the irreducible components of its exceptional divisor $E$ by $\left(E_{u}\right)_{u \in \mathcal{V}}$. The weighted dual graph $\Gamma$ of the resolution has $\mathcal{V}$ as vertex set. There are no loops, but as many edges between the distinct vertices $u, v$ as the intersection number $E_{u} \cdot E_{v}$. Moreover, each vertex $v \in \mathcal{V}$ is weighted by the self-intersection number $E_{v} \cdot E_{v}$ of $E_{v}$ on $\tilde{S}$.

Usually one decorates each vertex $u$ also by the genus of the corresponding component $E_{u}$. We do not do this, because those genera play no role in our study.

The set $\mathcal{V}$ may be seen not only as the vertex set of the dual graph $\Gamma$, but also as a set of parameters for canonical bases of the two following dual lattices, introduced by Lipman in [35, Section 18]:

$$
\Lambda:=\bigoplus_{u \in \mathcal{V}} \mathbb{Z} E_{u}, \check{\Lambda}:=\operatorname{Hom}_{\mathbb{Z}}(\Lambda, \mathbb{Z}) .
$$

The intersection form:

$$
I: \begin{array}{ccc}
\Lambda \times \Lambda & \rightarrow & \mathbb{Z} \\
\left(D_{1}, D_{2}\right) & \rightarrow & D_{1} \cdot D_{2}
\end{array}
$$

is the symmetric bilinear form on $\Lambda$ which computes the intersection number of compact divisors on $\tilde{S}$. The following fundamental theorem was proved by Du Val [16, Section 4] and Mumford [36, Page 6]. It is also a consequence of Zariski [48, Lemma 7.4]. See also a proof in Lipman [35, Lemma 14.1], where it is explained that the theorem remains true for normal surface singularities defined over arbitrary algebraically closed fields, possibly of positive characteristic:

Theorem 3. The intersection form of any resolution of $S$ is negative definite.

As a consequence, the map:

$$
\widetilde{I}: \Lambda \rightarrow \check{\Lambda}
$$

induced by the intersection form $I$ is an embedding of lattices, which allows to see $\Lambda$ as a sublattice of finite index of $\check{\Lambda}$, and $\check{\Lambda}$ as a lattice of the $\mathbb{Q}$-vector space $\Lambda_{\mathbb{Q}}:=\Lambda \otimes_{\mathbb{Z}} \mathbb{Q}$. In particular, the real vector space $\check{\Lambda}_{\mathbb{R}}$ gets identified with $\Lambda_{\mathbb{R}}$. As the intersection form $I$ extends canonically to $\Lambda_{\mathbb{Q}}$, one may restrict it to $\check{\Lambda}$. We will denote those extensions by the same symbol $I$. The following lemma is immediate:

Lemma 4. Seen as a subset of $\Lambda_{\mathbb{Q}}$, the lattice $\check{\Lambda}$ may be characterized in the following way:

$$
\check{\Lambda}=\left\{D \in \Lambda_{\mathbb{Q}} \mid D \cdot E_{u} \in \mathbb{Z}, \text { for all } u \in \mathcal{V}\right\} \text {. }
$$

Denote by $\delta_{u v}$ the Kronecker delta. The basis of $\check{\Lambda}$ which is dual to the basis $\left(E_{u}\right)_{u \in \mathcal{V}}$ of $\Lambda$ may be characterized in the following way as a subset of $\Lambda_{\mathbb{Q}}$ :

Definition 5. The Lipman basis of $\pi$ is the set $\left(\check{E}_{u}\right)_{u \in \mathcal{V}}$ of elements of the lattice $\check{\Lambda}$ defined by:

$$
\check{E}_{u} \cdot E_{v}=\delta_{u v} \text {, for all } v \in \mathcal{V} \text {. }
$$


Definition [5] implies immediately that:

$$
E_{u}=\sum_{w \in \mathcal{V}}\left(E_{w} \cdot E_{u}\right) \check{E}_{w}, \text { for all } u \in \mathcal{V}
$$

and that, conversely:

$$
\check{E}_{u}=\sum_{w \in \mathcal{V}}\left(\check{E}_{w} \cdot \check{E}_{u}\right) E_{w}, \text { for all } u \in \mathcal{V} .
$$

More generally, whenever $D \in \Lambda_{\mathbb{R}}=\check{\Lambda}_{\mathbb{R}}$, one has:

$$
D=\sum_{w \in \mathcal{V}}\left(D \cdot \check{E}_{w}\right) E_{w}=\sum_{w \in \mathcal{V}}\left(D \cdot E_{w}\right) \check{E}_{w} .
$$

The following result is well-known and goes back at least to Zariski [48, Lemma 7.1] (see it also creeping inside Artin's proof of [2, Prop. 2 (i)]):

Proposition 6. If $D \in \Lambda_{\mathbb{Q}} \backslash\{0\}$ is such that $D \cdot E_{v} \geq 0$ for all $v \in \mathcal{V}$, then all the coefficients $D \cdot \check{E}_{v}$ of $D$ in the basis $\left(E_{v}\right)_{v \in \mathcal{V}}$ of $\Lambda_{\mathbb{Q}}$ are negative.

Combining the equations (11) and (2), one gets:

Proposition 7. Once one chooses a total order on $\mathcal{V}$, the matrices $\left(E_{u} \cdot E_{v}\right)_{(u, v) \in \mathcal{V}^{2}}$ and $\left(\check{E}_{u} \cdot \check{E}_{v}\right)_{(u, v) \in \mathcal{V}^{2}}$ are inverse of each other.

By abuse of language, we say that the two previous functions from $\mathcal{V}^{2}$ to $\mathbb{Q}$ are matrices, even without a choice of total order on the index set $\mathcal{V}$. The intersection matrix $\left(E_{u} \cdot E_{v}\right)_{(u, v) \in \mathcal{V}^{2}}$ has negative entries on the diagonal (as a consequence of Theorem 3 ) and non-negative entries outside it. By contrast:

Proposition 8. The inverse matrix $\left(\check{E}_{u} \cdot \check{E}_{v}\right)_{(u, v) \in \mathcal{V}^{2}}$ has all its entries negative.

Proof. By formula (2), the entries of this matrix are the coefficients of the various rational divisors $\check{E}_{u}$ in the basis $\left(E_{v}\right)_{v \in \mathcal{V}}$ of $\Lambda_{\mathbb{Q}}$. By Definition [5] we see that $\check{E}_{u} \cdot E_{v} \geq 0$ for all $v \in \mathcal{V}$. One may conclude using Proposition 6

The fact that if the entries of a symmetric positive definite matrix are non-positive outside the diagonal, then the entries of the inverse matrix are non-negative was proved by Coxeter [11, Lemma 9.1] and differently by Du Val [15, Page 309], following a suggestion of Mahler. The stronger fact that in our case the entries of $\left(-\check{E}_{u} \cdot \check{E}_{v}\right)_{(u, v) \in \mathcal{V}^{2}}$ are positive comes from the fact that the dual graph of the initial matrix $\left(-E_{u} \cdot E_{v}\right)_{(u, v) \in \mathcal{V}^{2}}$ is connected. For historical details about this theme, see Coxeter [12, Section 10.9].

Proposition [ 6 may be reformulated as the fact that the pointed nef cone of $\pi$ is included in the interior of the opposite of the effective cone of $\pi$, where we use the following terminology, which is standard for global algebraic varieties:

Definition 9. Let $\pi$ be a resolution of $S$. The effective cone $\sigma$ of $\pi$ is the simplicial subcone of $\Lambda_{\mathbb{R}}$ consisting of those divisors with non-negative coefficients in the basis $\left(E_{u}\right)_{u \in \mathcal{V}}$. The nef cone $\check{\sigma}$ of $\pi$ is the simplicial subcone of $\check{\Lambda}_{\mathbb{R}}$, identified to $\Lambda_{\mathbb{R}}$ through $\tilde{I}$, consisting of those divisors whose intersections with all effective divisors are non-negative. That is, $\sigma$ is the convex cone generated by $\left(E_{u}\right)_{u \in \mathcal{V}}$ and $\check{\sigma}$ is the convex cone generated by $\left(\check{E}_{u}\right)_{u \in \mathcal{V}}$.

The determinant of a symmetric bilinear form on a lattice is well-defined: it is independent of the basis of the lattice used to compute it. When the bilinear form is positive definite, the determinant is positive. This motivates to look also at the opposite of the intersection form (see Neumann and Wahl [39, Sect. 12]). Up to the sign, the following notion was also studied in [18] and [38].

Definition 10. Let $\pi$ be a good resolution of $S$ with weighted dual graph $\Gamma$. The $\operatorname{determinant} \operatorname{det}(\Gamma) \in$ $\mathbb{N}^{*}$ of $\Gamma$ is the determinant of the opposite $-I$ of the intersection form, that is, the determinant of the matrix $\left(-E_{u} \cdot E_{v}\right)_{(u, v) \in \mathcal{V}^{2}}$. 
It is well-known (see for instance [13, Prop. 3.4 of Chap. 2]) that $\operatorname{det}(\Gamma)$ is equal to the cardinal of the torsion subgroup of the first integral homology group of the boundary (or link) of $S$. This shows that $\operatorname{det}(\Gamma)$ is independent of the choice of resolution of $S$. This fact could have been proved directly, by studying the effect of a blow-up of one point on the exceptional divisor of a given resolution and by using the fact that any two resolutions are related by a finite sequence of blow ups of points and of their inverse blow-downs. As a consequence, we define:

Definition 11. The determinant $\operatorname{det}(S)$ of the singularity $S$ is the determinant of the weighted dual graph of any good resolution of it.

\subsection{Mumford's rational intersection number of branches.}

As explained in the introduction, we will be mainly interested by the branches living on $S$ :

Definition 12. A branch on $S$ is a germ of irreducible formal curve on $S$. We denote by $\mathcal{B}(S)$ the set of branches on $S$. A divisor (respectively $\mathbb{Q}$-divisor) on $S$ is an element of the free abelian group (respectively $\mathbb{Q}$-vector space) generated by the branches living on it. The divisor is effective if all its coefficients are non-negative. It is principal if it is the divisor of a germ of formal function on $S$.

Note that the divisors we consider are Weil divisors, as they are not necessarily principal.

We will study the divisors on $S$ using their embedded resolutions, to which one extends the notion of weighted dual graph:

Definition 13. If $D$ is a divisor on $S$, an embedded resolution of $D$ is a resolution $\pi: \tilde{S} \rightarrow S$ of $S$ such that the preimage $\pi^{-1}|D|$ of $|D|$ on $\tilde{S}$ has simple normal crossings (here $|D|$ denotes the support of $D$ ). The weighted dual graph $\Gamma_{D}$ of $D$ with respect to $\pi$ is obtained from the weighted dual graph $\Gamma$ of $\pi$ by adding as new vertices the branches of $D$ and by joining each such branch $A$ to the unique vertex $u(A) \in \mathcal{V}$ such that $E_{u(A)}$ meets $A$.

The following construction of Mumford [36] of a canonical, possibly non-reduced structure of $\mathbb{Q}$-divisor on $\pi^{-1}|D|$, will be very important for us:

Definition 14. Let $A$ be a divisor on $S$ and $\pi: \tilde{S} \rightarrow S$ a resolution of $S$. The total transform of $A$ on $\tilde{S}$ is the $\mathbb{Q}$-divisor $\pi^{*} A=\tilde{A}+\left(\pi^{*} A\right)_{\text {ex }}$ on $\tilde{S}$ such that:

(1) $\tilde{A}$ is the strict transform of $A$ on $\tilde{S}$ (that is, the sum of the closures inside $\tilde{S}$ of the branches of $A$, keeping unchanged the coefficient of each branch).

(2) The support of the exceptional transform $\left(\pi^{*} A\right)_{e x}$ of $A$ on $\tilde{S}$ (or by $\pi$ ) is included in the exceptional divisor $E$.

(3) $\pi^{*} A \cdot E_{u}=0$ for each irreducible component $E_{u}$ of $E$.

Such a divisor $\left(\pi^{*} A\right)_{e x}$ exists and is unique:

Proposition 15. Let $A$ be a divisor on $S$ and $\pi: \tilde{S} \rightarrow S$ a resolution of $S$. Then the exceptional transform $\left(\pi^{*} A\right)_{\text {ex }}$ of $A$ is given by the following formula:

$$
\left(\pi^{*} A\right)_{e x}=-\sum_{u \in \mathcal{V}}\left(\tilde{A} \cdot E_{u}\right) \check{E}_{u} .
$$

In particular, $\left(\pi^{*} A\right)_{e x}$ lies in the opposite of the nef cone.

Proof. The third condition of Definition 14 implies that: $\left(\pi^{*} A\right)_{e x} \cdot E_{u}=-\tilde{A} \cdot E_{u}$, for all $u \in \mathcal{V}$. By combining this with equation (3), we get:

$$
\left(\pi^{*} A\right)_{e x}=\sum_{u \in \mathcal{V}}\left(\left(\pi^{*} A\right)_{e x} \cdot E_{u}\right) \check{E}_{u}=-\sum_{u \in \mathcal{V}}\left(\tilde{A} \cdot E_{u}\right) \check{E}_{u} .
$$

As $\tilde{A} \cdot E_{u} \geq 0$ for all $u \in \mathcal{V}$, we see that $-\left(\pi^{*} A\right)_{e x}$ lies in the cone generated by $\left(\check{E}_{u}\right)_{u \in \mathcal{V}}$ inside $\Lambda_{\mathbb{R}}$ which, by Definition 9 , is the nef cone $\check{\sigma}$. 
In the case in which $A$ is principal, defined by a germ of holomorphic function $f_{A}$, then $\left(\pi^{*} A\right)_{e x}$ is simply the exceptional part of the principal divisor on $\tilde{S}$ defined by the pull-back function $\pi^{*} f_{A}$. By Proposition 15, in this case $\left(\pi^{*} A\right)_{e x}$ belongs to the semigroup $-\check{\sigma} \cap \Lambda$ of integral exceptional divisors whose opposites are nef. In general not all the elements of this semigroup consist in such exceptional transforms of principal divisors, but this is true for those lying in the interior of $-\check{\sigma}$ :

Proposition 16. Consider a resolution of $S$. Any element of the lattice $\Lambda$ which lies in the interior of the cone $-\check{\sigma}$ has a multiple by a positive integer which is the exceptional transform of an effective principal divisor on $S$.

Proof. Denote by $K$ a canonical divisor on the resolution $\tilde{S}$ and by $E=\sum_{v \in \mathcal{V}} E_{v}$ the reduced exceptional divisor. By [9, Theorem 4.1], any divisor $D \in \Lambda$ such that:

$$
(D+E+K) \cdot E_{u} \leq-2 \text { for all } u \in \mathcal{V}
$$

is the exceptional transform of an effective principal divisor.

Assume now that $H \in \Lambda$ belongs to the interior of the opposite $-\check{\sigma}$ of the nef cone. This means that $H \cdot E_{u}<0$ for any $u \in \mathcal{V}$. There exists therefore $n \in \mathbb{N}^{*}$ such that: $n H \cdot E_{u}<-(E+K) \cdot E_{u}-2$, for all $u \in \mathcal{V}$. Equivalently, $D:=n H$ satisfies the inequalities (4), therefore it is the exceptional transform of an effective principal divisor.

Definition 14 allowed Mumford to introduce a rational intersection number of any two divisors on $S$ without common branches:

Definition 17. Let $A, B$ be two divisors on $S$ without common branches. Then their intersection number $A \cdot B \in \mathbb{Q}$ is defined by: $A \cdot B:=\pi^{*} A \cdot \pi^{*} B$, for any embedded resolution $\pi$ of $A+B$.

The fact that this definition is independent of the resolution was proved by Mumford [36, Section II (b)], by showing that it is unchanged if one blows up one point on $E$. As an immediate consequence we have:

Proposition 18. Let $A, B$ be two divisors on $S$ without common branches and $\left(\pi^{*} A\right)_{e x},\left(\pi^{*} A\right)_{e x}$ be their exceptional transforms on an embedded resolution $\tilde{S}$ of $A+B$. Then $A \cdot B=-\left(\pi^{*} A\right)_{e x} \cdot\left(\pi^{*} B\right)_{e x}$.

Assume now that $A$ is a branch on $S$. Consider an embedded resolution of it. Recall from Definition 13 that $u(A)$ denotes the unique index $u \in \mathcal{V}$ such that the strict transform $\tilde{A}$ meets $E_{u}$. Then one has another immediate consequence of the definitions:

Lemma 19. If $A$ is a branch on $S$ and if $\tilde{S}$ is an embedded resolution of $A$, then $\left(\pi^{*} A\right)_{e x}=-\check{E}_{u(A)}$.

By combining Proposition [18, Lemma 19] and Proposition 8, one gets:

\section{Corollary 20.}

(1) Assume that $A$ and $B$ are distinct branches on $S$. If $\tilde{S}$ is an embedded resolution of $A+B$, then:

$$
A \cdot B=-\check{E}_{u(A)} \cdot \check{E}_{u(B)}>0 \text {. }
$$

(2) If $E_{u}, E_{v}$ are two components of the exceptional divisor of a resolution, then the intersection number $\check{E}_{u} \cdot \check{E}_{v}$ is independent of the resolution (that is, it will be the same if one replaces $E_{u}, E_{v}$ by their strict transforms on another resolution).

\section{Generalities on ultrametrics and trees}

In this section we explain basic relations between ultrametrics, arborescent posets, hierarchies on finite sets, rooted and unrooted trees, height and depth functions on rooted trees, and additive distances on unrooted ones. The fact that we couldn't find these relations described in the literature in a way adapted to our purposes, explains the level of detail of this section. The framework developed here allows us to formulate in the next two sections simple conceptual proofs of our main results: Theorems 85, 87, 92, 119 and Corollary 120.

We begin by explaning the relation between rooted trees, arborescent posets and hierarchies (see Subsection 3.1). In Subsection 3.2 we recall the notion of ultrametric spaces and we explain that ultrametrics 
on finite sets may be alternatively presented as rooted trees endowed with a depth function, the intermediate object in this structural metamorphosis being the hierarchy of closed balls of the metric, which is an arborescent poset. We also define a dual notion of height function on a tree. This allows us to relate in Subsection 3.3 ultrametrics with additive distances on unrooted trees: we explain that any choice of root allows to transform canonically such a distance into a height function. Finally, in Subsection 4.3 we apply the previous considerations by giving a new proof of Płoski's original theorem, using the notion of Eggers-Wall tree.

\subsection{Trees, rooted trees, arborescent partial orders and hierarchies.}

If $V$ is a set, we denote by $\left(\begin{array}{c}V \\ 2\end{array}\right)$ the set of its subsets with 2 elements. There are two related notions of trees: combinatorial and topological.

Definition 21. A combinatorial tree is a finite combinatorial connected graph without cycles, that is, a pair $(\mathcal{V}, \mathcal{A})$ where $\mathcal{V}$ is a finite set of vertices, $\mathcal{A} \subset\left(\begin{array}{l}\mathcal{V} \\ 2\end{array}\right)$ is the set of edges and for any two distinct vertices $u, v$, there is a unique sequence $\left\{v_{0}, v_{1}\right\}, \ldots,\left\{v_{k-1}, v_{k}\right\}$ of edges, with $v_{0}=u, v_{k}=v$ and $v_{0}, \ldots, v_{k}$ pairwise distinct. Its geometric realization is the simplicial complex obtained by joining the vertices which are end-points of edges by segments in the real vector space $\mathbb{R}^{\mathcal{V}}$.

Definition 22. A topological tree $T$ is a topological space homeomorphic to the geometric realization of a combinatorial tree. If $u, v$ are two points of it, the unique embedded arc joining $u$ and $v$ is called the geodesic joining $u$ and $v$ and is denoted $[u v]=[v u]$. More generally, the convex hull $[V]$ of a finite subset $V$ of $T$ is the union of the geodesics joining pairwise the points of $V$. If $V=\{u, v, w, \ldots\}$, we denote also $[V]=[u v w . .$.$] . The valency of a point u \in T$ is the number of connected components of its complement $T \backslash\{u\}$. The ends of the topological tree are its points of valency 1 and its nodes are its points of valency at least 3. An underlying combinatorial tree being fixed (which will always be the case in the sequel), its vertices are by definition the vertices of $T$. A point $u \in T$ is called interior if it is not an end. Denote:

- $\mathcal{A}(T)=$ the set of edges of $T$;

- $\mathcal{V}(T)=$ the set of vertices of $T$;

- $\mathcal{E}(T)=$ the set of ends of $T$;

- $\mathcal{N}(T)=$ the set of nodes of $T$;

- $\mathcal{I}(T)=$ the set of interior vertices of $T$.

One has the inclusion $\mathcal{N}(T) \cup \mathcal{E}(T) \subset \mathcal{V}(T)$, which is strict if and only if either there is at least one vertex of valency 2 or the tree is reduced to a point (that is, $\mathcal{V}(T)$ has only one element, hence $\mathcal{A}(T)=\emptyset)$.

We will use also the following vocabulary about posets:

Definition 23. In a poset $(V, \preceq)$, we say that $u \in V$ is a predecessor of $v \in V$ or that $v$ is a successor of $u$ if $u \prec v$ (which means that the inequality is strict). We say that the predecessor $u$ of $v$ is a direct predecessor of $v$ if it is not a predecessor of any other predecessor of $v$. Then we say also that $v$ is a direct successor of $u$. Two elements of $V$ are comparable if one is a predecessor of the other, and directly comparable if one is a direct predecessor of the other one.

In the sequel we will work also with rooted trees, where the root may be either a vertex or a point interior to some edge. A choice of root of a given tree endows it with a canonical partial order:

Definition 24. Let $T$ be a topological tree. One says that it is rooted if a point $\rho \in T$ is chosen, called the root. Whenever we want to emphasize the root, we denote by $T_{\rho}$ the tree $T$ rooted at $\rho$. The associated partial order $\preceq_{\rho}$ on $T_{\rho}$ is defined by:

$$
u \preceq_{\rho} v \Longleftrightarrow[\rho u] \subset[\rho v] .
$$

The maximal elements for this partial order are called the leaves of $T_{\rho}$. Their set is denoted by $\mathcal{L}\left(T_{\rho}\right)$. The topological vertex set $\mathcal{V}_{t o p}\left(T_{\rho}\right)$ of $T_{\rho}$ consists of its leaves, its nodes and its root. That is, it is defined by:

$$
\mathcal{V}_{\text {top }}\left(T_{\rho}\right):=\mathcal{L}\left(T_{\rho}\right) \cup \mathcal{N}(T) \cup\{\rho\} .
$$




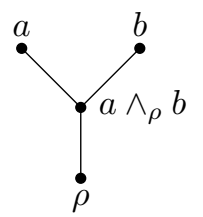

FIGURE 1. The infimum of $a$ and $b$ for the partial order $\preceq \rho$ (see Notation 25).

If $V$ is a finite set, the restriction to $V$ of a partial order $\preceq$ coming from a structure of rooted tree with vertex set containing $V$ is called an arborescent partial order. In this case, $(V, \preceq)$ is called an arborescent poset.

It is immediate to see that for a rooted tree $T_{\rho}$, the root $\rho$ is the absolute minimum of $\left(T_{\rho}, \preceq_{\rho}\right)$. In addition, the partial order $\preceq_{\rho}$ has well-defined infima of pairs of points. This motivates (see Figure 1):

Notation 25. Let $T_{\rho}$ be a rooted tree. The infimum of $a$ and $b$ for the partial order $\preceq_{\rho}$, that is, the maximal element of $[\rho a] \cap[\rho b]$, is denoted $a \wedge_{\rho} b$.

It will be important in the sequel to distinguish the trees in which the root is an end (which implies, by Definition 22, that the tree has at least two vertices):

Definition 26. An end-rooted tree is a rooted tree $T_{\rho}$ whose root is an end. Then the root $\rho$ has a unique direct successor $\rho^{+}$and each leaf $a$ has a unique direct predecessor $a^{-}$. The core $\stackrel{\circ}{T}_{\rho}$ of the end-rooted tree $T_{\rho}$ is the convex hull of $\left\{\rho^{+}\right\} \cup\left\{a^{-}, a \in \mathcal{L}\left(T_{\rho}\right)\right\}$, seen as a tree rooted at $\rho^{+}$.

A rooted tree which is not end-rooted, that is, such that the root is interior, is called interior-rooted.

Given an arbitrary rooted tree, there is a canonical way to embed it in an end-rooted tree:

Definition 27. Let $T_{\rho}$ be a tree rooted at $\rho$. Its extension $\hat{T}_{\hat{\rho}}$ is the tree obtained from $T_{\rho}$ by adding a new root $\hat{\rho}$, which is joined by an edge to $\rho$.

We defined arborescent posets starting from arbitrary rooted trees. Conversely, any arborescent poset $(V, \preceq)$ has a canonically associated rooted tree $T(V, \preceq)$ endowed with an underlying combinatorial tree. The root $\rho$ may not belong to $V$, but the vertex set of $T(V, \preceq)$ is exactly $V \cup\{\rho\}$. More precisely:

Definition 28. Let $(V, \preceq)$ be an arborescent poset. Its associated rooted tree $T(V, \preceq)$ is defined by:

- If $(V, \preceq)$ has a unique minimal element, then the root coincides with it, and the edges are exactly the sets of the form $\{u, v\}$, where $v$ is a direct predecessor of $u$. That is, $T(V, \preceq)$ is the underlying tree of the Hasse diagram of the poset.

- If $(V, \preceq)$ has several minimal elements, then one considers a new set $\hat{V}:=V \sqcup\{m\}$ and one extends the order $\preceq$ to it by imposing that $m$ is a predecessor of all the elements of $V$. Then one proceeds as in the previous case, working with $(\hat{V}, \preceq)$ instead of $(V, \preceq)$. In particular, the root is the new vertex $m$.

The extended rooted tree $\hat{T}(V, \preceq)$ of $(V, \preceq)$ is the extension of $T(V, \preceq)$ according to Definition 27

The fact that the objects introduced in Definition 28 are always trees is a consequence of the following elementary proposition, whose proof we leave to the reader:

Proposition 29. A partial order $\preceq$ on the finite set $V$ is arborescent if and only if any element of it has at most one direct predecessor.

The notion of extended rooted tree of an arborescent poset will play an important role in our context (see Remark 88).

Remark 30. We took the name of arborescent partial order from [19. The characterization given in Proposition 29] was chosen in [19] as the definition of this notion. 


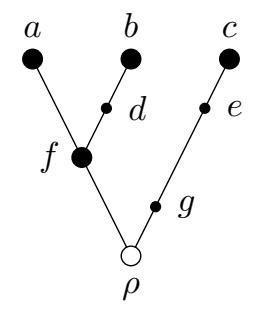

FigurE 2. The vertex set versus the topological vertex set in Example 33
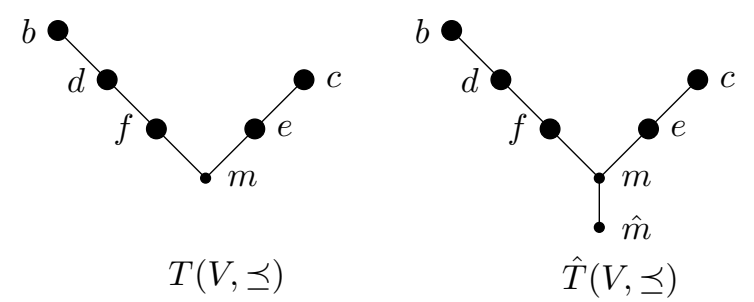

Figure 3. The two trees associated canonically to the arborescent poset $V$ of Example 33.

One has the following fact, whose proof we leave to the reader:

Proposition 31. Let $T_{\rho}$ be a rooted tree. Then the rooted tree $T\left(\mathcal{V}_{\text {top }}\left(T_{\rho}\right), \preceq_{\rho}\right)$ associated to the arborescent poset $\left(\mathcal{V}_{\text {top }}\left(T_{\rho}\right), \preceq \rho\right)$ is isomorphic to $T_{\rho}$ by an isomorphism which fixes $\mathcal{V}_{\text {top }}\left(T_{\rho}\right)$.

A rooted tree may also be encoded as a supplementary structure on its set of leaves. Namely:

Definition 32. Let $T_{\rho}$ be a rooted tree. To each point $v \in T_{\rho}$, associate its cluster $\mathbb{K}_{\rho}(v)$ as the set of leaves which have $v$ as predecessor:

$$
\mathbb{K}_{\rho}(v):=\left\{u \in \mathcal{L}\left(T_{\rho}\right), v \preceq_{\rho} u\right\} .
$$

Example 33. Figure 2 shows a rooted tree $T$ with vertex set $\{a, \ldots, g\}$ indicated by black bullets. It is rooted at a point $\rho$ which is not a vertex, indicated by a white bullet. Note that $\rho$ lies in the interior of the edge $[f g]$. The topological vertex set of $T$, indicated with bigger bullets, is $\mathcal{V}_{\text {top }}\left(T_{\rho}\right)=\{a, b, c, f, \rho\}$. The arborescent poset $\left(V=\{b, c, d, e, f\}, \preceq_{\rho}\right.$ ) (which is taken distinct from the vertex set, see Definition 24), may be described by the strict inequalities, $f \prec_{\rho} d \prec_{\rho} b$ and $e \prec_{\rho} c$, between directly comparable elements of $V$. Notice that the poset $V$ has two minimal elements $e$ and $f$. The root $m$ of the tree $T(V, \preceq)$ is a new point, since we are in the second case of Definition 28. The two rooted trees $T(V, \preceq)$ and $\hat{T}(V, \preceq)$ are drawn in Figure 3 In both cases, the vertices corresponding to the elements of $V$ are represented with bigger bullets. The clusters associated to the vertices and the root of $T_{\rho}$ are:

$$
\begin{gathered}
\mathbb{K}_{\rho}(a)=\{a\}, \mathbb{K}_{\rho}(b)=\mathbb{K}_{\rho}(d)=\{b\}, \mathbb{K}_{\rho}(f)=\{a, b\}, \\
\mathbb{K}_{\rho}(c)=\mathbb{K}_{\rho}(e)=\mathbb{K}_{\rho}(g)=\{c\}, \mathbb{K}_{\rho}(\rho)=\{a, b, c\} .
\end{gathered}
$$

One has the following direct consequences of Definition 32 .

\section{Proposition 34.}

(1) The cluster of a leaf $u$ is $\{u\}$ and the cluster of the root is the entire set of leaves $\mathcal{L}\left(T_{\rho}\right)$.

(2) The clusters $\mathbb{K}_{\rho}(u)$ and $\mathbb{K}_{\rho}(v)$ are disjoint if and only if $u$ and $v$ are incomparable.

(3) One has $\mathbb{K}_{\rho}(v) \subseteq \mathbb{K}_{\rho}(u)$ if and only if $u$ is a predecessor of $v$.

(4) Two points $u, v \in T$ have the same cluster if and only if one is a predecessor of the other one and the geodesic $[u v]$ does not contain nodes of $T_{\rho}$.

Denote by $2^{W}$ the power set of a set $W$, that is, its set of subsets. As an immediate consequence of Proposition 34 one has (recall that $\mathcal{V}_{\text {top }}\left(T_{\rho}\right)$ denotes the topological vertex set of $T_{\rho}$, introduced in Definition 24): 
Corollary 35. The cluster map:

$$
\begin{array}{ccc}
\mathbb{K}_{\rho}: \mathcal{V}_{\text {top }}\left(T_{\rho}\right) & \rightarrow 2^{\mathcal{L}\left(T_{\rho}\right)} \\
u & \rightarrow \mathbb{K}_{\rho}(u)
\end{array}
$$

is decreasing from the poset $\left(\mathcal{V}_{\text {top }}\left(T_{\rho}\right), \preceq_{\rho}\right)$ to the poset $\left(2^{\mathcal{L}\left(T_{\rho}\right)}, \subseteq\right)$. Moreover:

(1) If $T_{\rho}$ is not end-rooted, then $\mathbb{K}_{\rho}$ is injective.

(2) If $T_{\rho}$ is end-rooted, then $\mathbb{K}_{\rho}$ is injective in restriction to $\mathcal{V}_{\text {top }}\left(T_{\rho}\right) \backslash\{\rho\}$ and $\mathbb{K}_{\rho}(\rho)=\mathbb{K}_{\rho}\left(\rho^{+}\right)$, where $\rho^{+}$is the unique direct successor of $\rho$ in the poset $\left(\mathcal{V}_{\text {top }}\left(T_{\rho}\right), \preceq_{\rho}\right)$.

Proposition 34 may be reformulated by saying that the image of the cluster map is a hierarchy in the following sense:

Definition 36. A hierarchy on the finite set $X$ is a subset of $2^{X} \backslash\{\emptyset\}$ (whose elements are called clusters) satisfying the following properties:

(1) All the one-element subsets of $X$ as well as $X$ itself are clusters.

(2) Given any two clusters, they are either disjoint or one is included inside the other.

In fact, those properties characterize completely the images of cluster maps associated to rooted trees with given leaf set (folklore, see [4, Introduction]):

Proposition 37. The images of the cluster maps associated to the rooted trees with finite sets of leaves $X$ are exactly the hierarchies on $X$.

Now, if one orders a hierarchy by reverse inclusion, one gets an arborescent poset:

Lemma 38. Let $\mathcal{H}$ be a hierarchy on the finite set $X$. Define the partial order $\preceq_{\text {ri }}$ on $\mathcal{H}$ by:

$$
K_{1} \preceq_{r i} K_{2} \Longleftrightarrow K_{1} \supseteq K_{2} .
$$

Then $\left(\mathcal{H}, \preceq_{r i}\right)$ is an arborescent poset.

Proof. By Proposition 29, it is enough to check that for any cluster $K_{1} \neq X$, there exists a unique cluster $K_{2}$ strictly containing it and such that there are no other clusters between $K_{1}$ and $K_{2}$. But this comes from the fact that, by condition (2) of Definition 36. all the clusters containing $K_{1}$ form a chain (that is, a totally ordered set) under inclusion.

Conversely, one has the following characterization of arborescent posets coming from hierarchies, whose proof we leave to the reader:

Proposition 39. An arborescent poset $(V, \preceq)$ is isomorphic to the poset defined by a hierarchy if and only if any non-maximal element has at least two direct successors. In particular, the associated rooted tree $T(V, \preceq)$ is never end-rooted.

\subsection{Ultrametric spaces and dated rooted trees.}

In this subsection we explain the relation between finite ultrametric spaces and rooted trees endowed with a depth function.

Let us first fix our notations and vocabulary about metric spaces:

Notation 40. If $(X, d)$ is a metric space, then a closed ball of it is a subset of the form: $\mathbb{B}(a, r):=\{p \in$ $X \mid d(a, p) \leq r\}$, where $a$ denotes any point of $X$ and $r \in[0,+\infty)$. Each time a subset of $X$ is presented in this way, one says that $a$ is a center and $r$ is a radius of it. The diameter of a subset $Y \subset X$ is $\operatorname{diam}(Y):=\sup \{d(x, y), x, y \in Y\} \in[0,+\infty]$.

In Euclidean geometry, a closed ball has a unique center and a unique radius. None of those two properties remains true in general. There is an extreme situation in which any point of a closed ball is a center of it: 
Definition 41. Let $(X, d)$ be a metric space. The distance function $d: X \times X \rightarrow[0, \infty)$ is called an ultrametric if one has the following strong form of the triangle inequality, called the ultrametric inequality:

$$
d(a, b) \leq \max \{d(a, c), d(b, c)\}, \text { for all } a, b, c \in X .
$$

In this case, we say that $(X, d)$ is an ultrametric space.

One has the following characterizations of ultrametricity, which result immediately from the definition:

Proposition 42. Let $(X, d)$ be a metric space. Then the following conditions are equivalent:

(1) $d$ is an ultrametric.

(2) All the triangles are either equilateral or isosceles with the unequal side being the shortest.

(3) For any closed ball, all its points are centers of it.

(4) Given any two closed balls, they are either disjoint, or one of them is contained into the other one.

As a consequence of Proposition 42, we have the following property:

Lemma 43. Let $(X, U)$ be a finite ultrametric space. If $\mathcal{D}$ is a closed ball, then its diameter $\operatorname{diam}(\mathcal{D})$ is the minimal radius $r$ such that $\mathcal{D}=\mathbb{B}(a, r)$ for any $a \in \mathcal{D}$.

The prototypical examples of ultrametric spaces are the fields of $p$-adic numbers or, more generally, all the fields endowed with a non-Archimedean norm. Our goal in the rest of this section is to describe the canonical presentation of finite ultrametric spaces as sets of leaves of finite rooted trees (see Proposition 50 below). A pleasant elementary introduction to this view-point is contained in Holly's paper 29.

The basic fact indicating that rooted trees are related with ultrametric spaces is the similarity of the conditions defining hierarchies (see Definition 36) with the characterization of ultrametrics given as point (41) of Proposition 42. This characterization, combined with the fact that the closed balls of radius 0 are exactly the subsets with one element, and the fact that on a finite metric space, the closed balls of sufficiently big radius are the whole set, shows that:

Lemma 44. The set $\mathcal{B}(X, U)$ of closed balls of a finite ultrametric space $(X, U)$ is a hierarchy on $X$.

As a consequence of Lemmas 38 and 44, an ultrametric $U$ on a finite set $X$ defines canonically two rooted trees with leaf-set $X$ and topological vertex set $\mathcal{B}(X, U)$ :

Definition 45. Let $U$ be an ultrametric on the finite set $X$. The interior-rooted tree $T^{U}$ associated to the ultrametric $U$ is the rooted tree $T\left(\mathcal{B}(X, U), \preceq_{r i}\right)$ determined by the arborescent poset of closed balls of $U$. The end-rooted tree $\hat{T}^{U}$ associated to the ultrametric $U$ is the extended rooted tree $\hat{T}\left(\mathcal{B}(X, U), \preceq_{r i}\right)$.

The terminology is motivated by Proposition 39, which implies that $T^{U}$ is indeed always interior-rooted and $\hat{T}^{U}$ always end-rooted. By Definition 28 and Lemma 38, the root $\rho$ of $T^{U}$ corresponds to the set $X$, while the root $\hat{\rho}$ of $\hat{T}^{U}$ is defined as the immediate predecessor of $\rho$ in the tree $\hat{T}^{U}$.

One may encode also the values of the metric $U$ on its end-rooted tree $\hat{T}^{U}$, as a decoration on its set $\mathcal{I}\left(\hat{T}^{U}\right)$ of interior vertices:

Definition 46. Let $(X, U)$ be a finite ultrametric space. Its diametral function:

$$
\delta^{U}: \mathcal{I}\left(\hat{T}^{U}\right) \rightarrow \mathbb{R}_{+}^{*},
$$

defined on the set of interior vertices of the end-rooted tree $\hat{T}^{U}$ of $(X, U)$, associates to each vertex $u \in \mathcal{I}\left(\hat{T}^{U}\right)$ the diameter of its cluster $\mathbb{K}_{\hat{\rho}}(u)$.

The root $\rho$ of $T^{U}$ is always an interior vertex of $\hat{T}^{U}$, and its diameter $\delta^{U}(\rho)$ is equal to the diameter of $X$. Notice also that $\mathbb{K}_{\rho}(u)=\mathbb{K}_{\hat{\rho}}(u)$, for all $u \in \mathcal{I}\left(\hat{T}^{U}\right)=\mathcal{I}\left(T^{U}\right)$.

The diametral function of a finite ultrametric space is a depth function on the associated end-rooted tree in the following sense: 
Definition 47. A depth function on a rooted tree $T_{\rho}$ is a strictly decreasing function:

$$
\delta:\left(\mathcal{I}\left(T_{\rho}\right), \preceq \rho\right) \rightarrow\left(\mathbb{R}_{+}^{*}, \leq\right)
$$

That is, $\delta(v)<\delta(u)$ whenever $u \prec_{\rho} v$. A pair $\left(T_{\rho}, \delta\right)$ of a rooted tree and a depth function on it is called a depth-dated tree.

Intuitively, such a function $\delta$ measures the depth of the interior vertices as seen from the leaves, if one imagines that the leaves are above the root, as modeled by the partial order $\preceq_{\rho}$.

We have explained how to pass from an ultrametric on a finite set to a depth-dated rooted tree (see Definitions 45 and 46). Conversely, given such a tree, one may construct an ultrametric on its set of leaves (recall that $a \wedge_{\rho} b$ is the infimum of $a$ and $b$ for the partial order $\preceq_{\rho}$, see Notation 25):

Lemma 48. Let $\left(T_{\rho}, \delta\right)$ be a depth-dated rooted tree. Then the function $U^{\delta}: \mathcal{L}\left(T_{\rho}\right) \times \mathcal{L}\left(T_{\rho}\right) \rightarrow \mathbb{R}_{+}$defined by:

is an ultrametric on $\mathcal{L}\left(T_{\rho}\right)$.

$$
U^{\delta}(a, b):= \begin{cases}\delta\left(a \wedge_{\rho} b\right) & \text { if } a \neq b \\ 0 & \text { if } a=b\end{cases}
$$

Proof. Consider $a, b, c \in \mathcal{L}\left(T_{\rho}\right)$. The inequality:

$$
U^{\delta}(a, b) \leq \max \left\{U^{\delta}(a, c), U^{\delta}(b, c)\right\}
$$

is equivalent to:

$$
\delta\left(a \wedge_{\rho} b\right) \leq \max \left\{\delta\left(a \wedge_{\rho} c\right), \delta\left(b \wedge_{\rho} c\right)\right\},
$$

which is in turn a consequence of the facts that $\delta$ is a depth function and that:

$$
a \wedge_{\rho} b \geq \min \left\{a \wedge_{\rho} c, b \wedge_{\rho} c\right\} .
$$

The previous inequality, including the existence of this minimum (taken relative to the rooted tree partial order $\prec_{\rho}$ ) is a basic property of rooted trees.

Moreover, if the tree $T_{\rho}$ is end-rooted, then one may reconstruct it as the end-rooted tree associated to the ultrametric space $\left(\mathcal{L}\left(T_{\rho}\right), U^{\delta}\right)$ :

Proposition 49. Let $\left(T_{\rho}, \delta\right)$ be a depth-dated end-rooted tree. There exists a unique isomorphism fixing the set of leaves between the combinatorial rooted trees underlying the depth-dated trees:

- $T_{\rho}$ endowed with the topological vertex set $\mathcal{V}_{\text {top }}\left(T_{\rho}\right)$ and with the restriction to its set of interior vertices of the depth function $\delta$;

- the depth-dated tree $\left(\hat{T}^{U^{\delta}}, \delta^{U^{\delta}}\right)$ associated to the ultrametric $U^{\delta}$.

Taken together, the previous considerations prove the announced bijective correspondence between ultrametrics on a finite set $X$ and a special type of depth-dated end-rooted trees with set of leaves $X$ :

Proposition 50. Let $X$ be a finite set. The map which associates to an ultrametric $U$ on $X$ the diametral function $\delta^{U}$ on the end-rooted tree $\hat{T}^{U}$ realizes a bijective correspondence between ultrametrics on $X$ and isomorphism classes of depth-dated end-rooted rooted trees $\left(T_{\rho}, \delta\right)$ with set of vertices equal to their topological vertex set and with set of leaves equal to $X$.

The following notion is dual to that of depth functions:

Definition 51. A height function on a rooted tree $T_{\rho}$ is a strictly increasing function:

$$
h:\left(\mathcal{I}\left(T_{\rho}\right), \preceq_{\rho}\right) \rightarrow\left(\mathbb{R}_{+}, \leq\right) .
$$

That is, $h(u)<h(v)$ whenever $u \prec_{\rho} v$. A pair $\left(T_{\rho}, h\right)$ of a rooted tree and a height function on it is called a height-dated tree.

Remark 52. Note the slight assymmetry of the two definitions: we impose that depth functions take positive values, but we allow a height function to vanish. This assymmetry is motivated by the fact that we use depth functions to define ultrametrics by Lemma 48. The condition of strict increase on a height function imposes that a vanishing may occur only at the minimal element of $\mathcal{I}\left(T_{\rho}\right)$, which is either the root $\rho$ (if $T_{\rho}$ is interior-rooted) or its immediate successor in $\mathcal{V}\left(T_{\rho}\right)$ (if $T_{\rho}$ is end-rooted). 


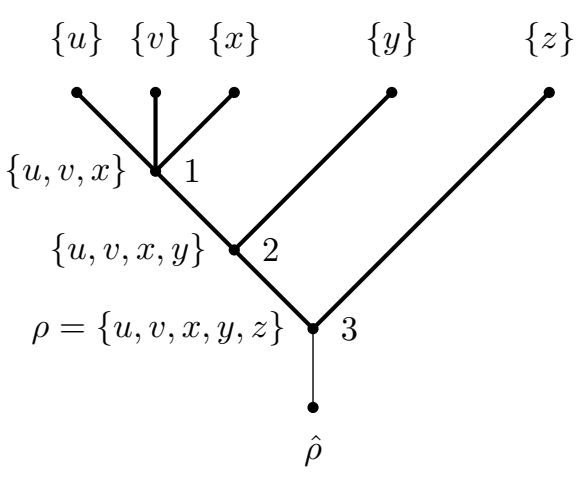

Figure 4. The depth-dated tree associated to the ultrametric of Example 55 .

Any strictly decreasing function allows to transform height functions into depth functions:

Lemma 53. Any strictly decreasing map:

$$
s:\left(\mathbb{R}_{+}, \leq\right) \rightarrow\left(\mathbb{R}_{+}^{*}, \leq\right)
$$

transforms by left-composition all height functions on a rooted tree into depth functions.

Remark 54. In [4, Böcker and Dress defined more general symbolically dating maps on trees, taking values in arbitrary sets, and characterize the associated symbolic ultrametrics by a list of axioms. We don't use here that generalized setting. Nevertheless we mention it because that paper inspired us in our work. For instance, we introduced the names depth-dated/height-dated tree by following its "dating" terminology (which seems standard in part of the mathematical literature concerned with problems of classifications, as mathematical phylogenetics).

Example 55. Consider a set $X=\{u, v, x, y, z\}$ and a function $U: X^{2} \rightarrow \mathbb{R}_{+}$whose matrix $(U(a, b))_{a, b \in X}$ is (for the order $u<\cdots<z$ ):

$$
\left(\begin{array}{lllll}
0 & 1 & 1 & 2 & 3 \\
1 & 0 & 1 & 2 & 3 \\
1 & 1 & 0 & 2 & 3 \\
2 & 2 & 2 & 0 & 3 \\
3 & 3 & 3 & 3 & 0
\end{array}\right) .
$$

It is immediate to check that $U$ is an ultrametric distance. The hierarchy of its closed balls is:

$$
\{\{u\}, \ldots,\{z\},\{u, v, x\},\{u, v, x, y\},\{u, v, x, y, z\}\} .
$$

The associated rooted trees $T^{U}$ and $\hat{T}^{U}$ are represented in Figure $4 . T^{U}$ being drawn with thicker segments. Near each vertex is written the associated cluster. Near each interior vertex $u$ of $\hat{T}^{U}$ is also written the value of the diametral function $\delta^{U}(u)$, that is, the diameter of the cluster $\mathbb{K}_{\rho}(u)$.

\subsection{Additive distances on trees.}

Let us pass now to the notion of additive distance on an unrooted tree. Our aim in this subsection is to explain in which way such a distance defines plenty of ultrametrics (see Proposition 62).

Definition 56. Let $(V, A)$ be a combinatorial tree. An additive distance on it is a symmetric map $d: V \times V \rightarrow \mathbb{R}_{+}$such that:

(1) $d(u, v)=0$ if and only if $u=v$;

(2) $d(u, v)+d(v, w)=d(u, w)$ whenever $v \in[u w]$.

Of course, the additive distance $d$ is a metric on $V$. Buneman $[6$ characterized such metrics in the following way: 

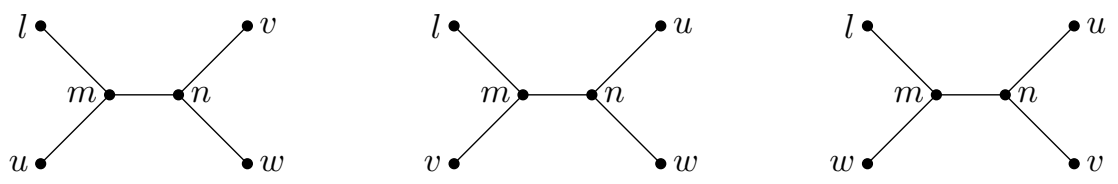

FiguRE 5. The three possible generic trees with four leaves.

Proposition 57. A metric $d$ on the finite set $V$ comes from an additive distance function on a combinatorial tree with vertex set $V$ if and only if it satisfies the following four points condition:

$$
d(l, u)+d(v, w) \leq \max \{d(l, v)+d(u, w), d(l, w)+d(u, v)\} \text { for any } l, u, v, w \in V .
$$

In fact, one has the following more precise inequality, which we leave as an exercise to the reader:

Proposition 58. Let $d$ be an additive distance on the finite combinatorial tree $(V, A)$. Consider also its geometric realization, inside which will be taken convex hulls. Then, for every $l, u, v, w \in V$, one has:

$$
d(l, v)+d(u, w) \geq d(l, u)+d(v, w) \Longleftrightarrow[l v] \cap[u w] \neq \emptyset,
$$

with equality if and only if one has moreover $[l u] \cap[v w] \neq \emptyset$, that is, if and only if the convex hull [luvw] is like in the right-most tree of Figure 5, or as in one of its degenerations.

More degenerate configurations are obtained by contracting to points one or more segments of Figure 5. For instance, it is possible to have $m=n$ or $m=l$, etc.

Assume now that $(T, d)$ is a topological tree endowed with an additive distance (on its underlying combinatorial tree). Choose a root $\rho \in \mathcal{V}(T)$. The distance function may now be encoded alternatively as a more general height function:

Definition 59. The remoteness function associated to the additive distance $d$ on the rooted tree $T_{\rho}$ is defined by:

$$
\begin{aligned}
& h_{d, \rho}: \mathcal{V}\left(T_{\rho}\right) \quad \rightarrow \quad \mathbb{R}_{+} \\
& u \rightarrow d(u, \rho) .
\end{aligned}
$$

Note that, as may be verified simply by looking at Figure 1, the remoteness function allows to reconstruct the additive distance:

Lemma 60. Assume that $d$ is an additive distance on the rooted tree $T_{\rho}$. Then one has the following equivalent equalities:

(1) $d(a, b)=h_{d, \rho}(a)+h_{d, \rho}(b)-2 h_{d, \rho}\left(a \wedge_{\rho} b\right)$ for all $a, b \in \mathcal{V}\left(T_{\rho}\right)$.

(2) $h_{d, \rho}\left(a \wedge_{\rho} b\right)=\frac{1}{2} \cdot(d(\rho, a)+d(\rho, b)-d(a, b))$ for all $a, b \in \mathcal{V}\left(T_{\rho}\right)$.

In the following results we consider an end-rooted tree $T_{\hat{\rho}}$ with non-empty core $\stackrel{\circ}{\rho}_{\rho}$ (see Definition 26). Notice that the set of vertices of the core $\stackrel{\circ}{T}_{\rho}$ is the set of interior vertices of $T_{\hat{\rho}}$ and that $\stackrel{\circ}{T}_{\rho}$ is considered as a rooted-tree, the root $\rho$ being the immediate successor of $\hat{\rho}$ in $T_{\hat{\rho}}$. It is immediate to see that:

Lemma 61. Consider an end-rooted tree $T_{\hat{\rho}}$ with non-empty core $\stackrel{\circ}{T}_{\rho}$. Then the map: $d \rightarrow h_{d, \rho}$, which associates to an additive distance function of $\stackrel{\circ}{T}_{\rho}$ its remoteness function, establishes a bijection from the set of additive distances on $\stackrel{\circ}{T}$ to the set of height functions of $T_{\hat{\rho}}$ which vanish at $\rho$.

Combining Lemmas 61, 53 and 48 , we get:

Proposition 62. Assume that $T_{\hat{\rho}}$ is an end-rooted tree with non-empty core $\stackrel{\circ}{T}_{\rho}$, which is endowed with an additive distance function $d$. Then, for any strictly decreasing function $s:\left(\mathbb{R}_{+}, \leq\right) \rightarrow\left(\mathbb{R}_{+}^{*}, \leq\right)$, the function $U^{d, \rho, s}: \mathcal{L}\left(T_{\hat{\rho}}\right) \times \mathcal{L}\left(T_{\hat{\rho}}\right) \rightarrow \mathbb{R}_{+}$defined by:

$$
U^{d, \rho, s}(a, b):= \begin{cases}s\left(h_{d, \rho}\left(a \wedge_{\rho} b\right)\right) & \text { if } a \neq b \\ 0 & \text { otherwise, }\end{cases}
$$

is an ultrametric on the set of leaves $\mathcal{L}\left(T_{\hat{\rho}}\right)$. 
Therefore, starting from an end-rooted tree whose core is endowed with an additive distance, one gets plenty of ultrametric spaces, depending on the choice of map s. Each such ultrametric space has two associated rooted trees, as explained in Definition 45. By Proposition 50, the end-rooted one may be identified topologically with the initial end-rooted tree:

Proposition 63. Let $T_{\hat{\rho}}$ be an end-rooted tree with non-empty core $\stackrel{\circ}{T}_{\rho}$. We assume that $\stackrel{\circ}{T}_{\rho}$ is endowed with an additive distance function $d$. Consider $T_{\hat{\rho}}$ as a combinatorial rooted tree with vertex set equal to its topological vertex set $\mathcal{V}_{\text {top }}\left(T_{\hat{\rho}}\right)$, as defined by (5). Let $s:\left(\mathbb{R}_{+}, \leq\right) \rightarrow\left(\mathbb{R}_{+}^{*}, \leq\right)$ be a strictly decreasing map and let $U^{d, \rho, s}: \mathcal{L}\left(T_{\hat{\rho}}\right) \times \mathcal{L}\left(T_{\hat{\rho}}\right) \rightarrow \mathbb{R}_{+}$be the ultrametric defined in Proposition 62. Then:

(1) The end-rooted tree $\hat{T}^{U^{d, \rho, s}}$ is uniquely isomorphic with $T_{\hat{\rho}}$ as a combinatorial rooted tree with leaf-set $\mathcal{L}\left(T_{\hat{\rho}}\right)$. In restriction to $\mathcal{I}\left(T_{\hat{\rho}}\right)$, this isomorphism identifies the diametral function $\delta^{U^{d, \rho, s}}$ of Definition 46 with $s \circ h_{d, \rho}$.

(2) The previous isomorphism identifies the interior-rooted tree $T^{U^{d, \rho, s}}$ with the convex hull $\left[\mathcal{L}\left(T_{\hat{\rho}}\right)\right]$, as rooted trees with leaf-set $\mathcal{L}\left(T_{\hat{\rho}}\right)$.

Proposition 63 is a special case of the theory explained by Böcker and Dress in [4].

\section{Arborescent singularities ANd their Ultrametric SPACES OF BRAnCheS}

In this section we introduce the notion of arborescent singularity and we prove that, given any good resolution of such a singularity, there is a natural additive metric on its dual tree, constructed from a determinantal identity of Eisenbud and Neumann. We deduce from this additivity the announced results about the fact that the functions $U_{L}$ defined in the introduction are ultrametrics, and their relation with the dual trees of resolutions.

\subsection{Determinant products for arborescent singularities.}

In this subsection we explain the basic facts about arborescent singularities needed in the sequel.

Definition 64. A normal surface singularity is called arborescent if the dual graph of some good resolution of it is a tree.

It is immediate to see, using the fact that there exists a minimal good resolution, which any good resolution dominates by a sequence of blow-ups of points, that the dual graph of some good resolution is a tree if and only if this is the case for any good resolution.

Remark 65. All normal quasi-homogeneous, rational, minimally elliptic singularities which are not cusp singularities or Neumann and Wahl's [39] splice quotient singularities are arborescent. Note that the first three classes of singularities are special cases of splice quotients whenever their boundaries are rational homology spheres (which is always the case for rational ones, but means that the quotient by the $\mathbb{C}^{*}$ action is a rational curve in the quasihomogeneous case, and that one does not have a simply elliptic singularity - a special case of quasihomogeneous singularities, in which this quotient is elliptic and there are no special orbits - in the minimally elliptic case). This was proved by Neumann 37 for the quasihomogeneous singularities and by Okuma [41] for the other classes (see also [39. Appendix]). Note that all splice quotients are special cases of normal surface singularities with rational homology sphere links, which in turn are all arborescent by [13, Proposition 3.4 of Chap. 2].

Remark 66. José Seade told us that arborescent singularities had also appeared, but without receiving a special name, in Camacho's paper [7].

The notions explained in the following definition were introduced with slightly different terminology by Eisenbud and Neumann [18, Sect. 20,21] (recall that the notion of determinant of a weighted graph was explained in Definition [10]):

Definition 67. Let $S$ be an arborescent singularity. Consider any good resolution of it. For each vertex $u$ of its dual tree $\Gamma$ and each edge $e$ containing $u$, we say that the subtree $\Gamma_{u, e}$ of $u$ in the direction $e$ is the full subtree of $\Gamma$ whose vertices are those vertices $t$ of $\Gamma$ distinct from $u$, which are seen from $u$ in the 


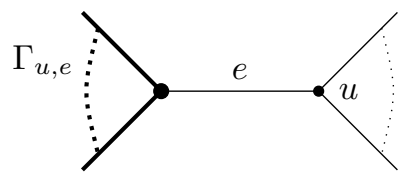

Figure 6. The subtree $\Gamma_{u, e}$ of Definition 67 is sketched with thicker segments.

direction of the edge $e$, that is, such that $e \subset[u t]$ (see Figure 6). The edge $\operatorname{determinant} \operatorname{det}_{u, e}(\Gamma)$ at the vertex $u$ in the direction $e$ is the determinant of $\Gamma_{u, e}$. For any $v, w \in \mathcal{V}$, define the determinant product $p(v, w)$ of the pair $(v, w)$ as the product of the determinants at all the points of the geodesic $[v w]$ which connects $v$ and $w$, in the directions of the edges which are not contained in that geodesic.

Note that the definition implies that $p(v, w)=p(w, v) \in \mathbb{N}^{*}$ for any $v, w \in \mathcal{V}$.

In order to compute determinant products in concrete examples, it is important to be able to compute rapidly determinants of weighted trees. One could use the general algorithms of linear algebra. Happily, there exists a special algorithm adapted to tree determinants, which was presented in Duchon's thesis [14, Sect. III.1] and studied in [18, Section 21]. We used it a lot for our experimentations. This algorithm may be formulated as follows:

Proposition 68. Let $\Gamma_{u}$ be the dual tree of a good resolution of an arborescent singularity, rooted at one of its vertices $u$. For any vertex $v$ of $\Gamma_{u}$, denote by $-\alpha_{v}<0$ the self-intersection of the component $E_{v}$. Denote also by $\left(e_{j}\right)_{j \in J(u)}$ the edges of $\Gamma_{u}$ containing $u$. Each subtree $\Gamma_{u, e_{j}}$ is considered to be rooted at the vertex of $e_{j}$ different from $u$. Define recursively the continued fraction $c f\left(\Gamma_{u}\right)$ of the weighted rooted tree $\Gamma_{u}$ by:

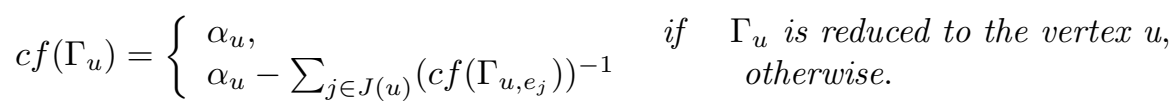

Then:

$$
\operatorname{det}(\Gamma)=c f\left(\Gamma_{u}\right) \cdot \prod_{j \in J(u)} \operatorname{det}\left(\Gamma_{u, e_{j}}\right) .
$$

The following multiplicative property of determinant products will be fundamental for us in the sequel:

Proposition 69. For any three vertices $u, v, w \in \mathcal{V}$ such that $v \in[u w]$, one has:

$$
p(u, v) \cdot p(v, w)=p(v, v) \cdot p(u, w) .
$$

Equivalently:

$$
\frac{p(u, v)}{\sqrt{p(u, u) \cdot p(v, v)}} \cdot \frac{p(v, w)}{\sqrt{p(v, v) \cdot p(w, w)}}=\frac{p(u, w)}{\sqrt{p(u, u) \cdot p(w, w)}} .
$$

Proof. The following proof is to be followed on Figure 7 We define:

- $P(u)=$ the product of edge determinants at the vertex $u$, over the set of edges starting from $u$ and not contained in $[u w]$. The products $P(v)$ and $P(w)$ are defined analogously.

- $P(u v)=$ the product of edge determinants at all vertices of $\Gamma$ situated in the interior of the geodesic $[u v]$, over the edges not contained in $[u w] . P(v w)$ is defined analogously.

- $M=$ the edge determinant at $v$ in the direction of the unique edge starting from $v$ and contained in $[u v] . N$ is defined analogously.

Then one has the following formulae, clearly understandable on Figure 7 ,

$$
\begin{array}{lll}
p(u, v)=P(u) \cdot P(u v) \cdot P(v) \cdot N, & p(v, w)=M \cdot P(v) \cdot P(v w) \cdot P(w), \\
p(v, v)=M \cdot N \cdot P(v), & p(u, w)=P(u) \cdot P(u v) \cdot P(v) \cdot P(v w) \cdot P(w) .
\end{array}
$$

The equality (6) is a direct consequence of the previous factorisations. Finally, it is immediate to see that (6) and (7) are equivalent. 


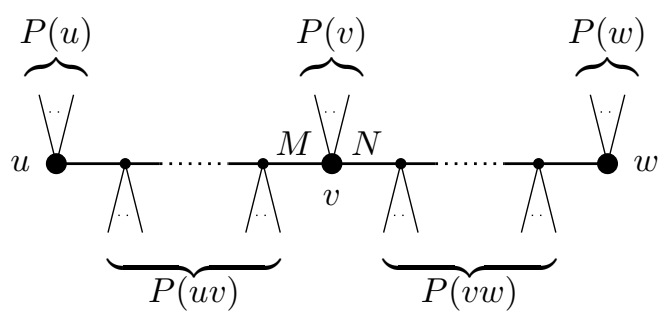

Figure 7. Illustration for the proof of Proposition 69.

The following proposition was proved by Eisenbud and Neumann [18, Lemma 20.2] (see also Neumann and Wahl [39, Theorem 12.2]) for trees corresponding to the singularities whose boundaries $\partial M$ are rational homology spheres. Nevertheless, their proofs use only the fact that those are weighted trees appearing as dual graphs of singularities.

Proposition 70. Let $S$ be an arborescent singularity. Consider any good resolution of it. Then, for any $v, w \in \mathcal{V}$, one has:

$$
p(v, w)=\left(-\check{E}_{v} \cdot \check{E}_{w}\right) \cdot \operatorname{det}(S) .
$$

Remark 71. Using Proposition [70, formula (6) becomes the equality:

$$
\left(-\check{E}_{u} \cdot \check{E}_{v}\right)\left(-\check{E}_{v} \cdot \check{E}_{w}\right)=\left(-\check{E}_{v} \cdot \check{E}_{v}\right)\left(-\check{E}_{u} \cdot \check{E}_{w}\right) \text {, for any } v \in[u w]
$$

of the introduction. After having seen a previous version of this paper, Jonsson told us that he had proved this equality for dual trees of compactifying divisors of $\mathbb{C}^{2}$ and Némethi told us that the equality could also be proved using Lemma 4.0.1 from his paper [5] written with Braun.

Remark 72. Let us consider the real vector space $\Lambda_{\mathbb{R}}=\check{\Lambda}_{\mathbb{R}}$ endowed with the opposite of the intersection form. It is a Euclidean vector space. Measuring the angles with this Euclidean metric, the equality (8) becomes:

$$
\cos \left(\angle \check{E}_{u} \check{E}_{v}\right) \cdot \cos \left(\angle \check{E}_{v} \check{E}_{w}\right)=\cos \left(\angle \check{E}_{u} \check{E}_{w}\right), \text { for any } v \in[u w]
$$

By the spherical pythagorean theorem, this means that the spherical triangle whose vertices are the unit vectors determined by $\check{E}_{u}, \check{E}_{v}, \check{E}_{w}$ is rectangle at the vertex corresponding to $\check{E}_{v}$. This interpretation was noticed by the third author in the announcement [4] of the main results of this paper.

Corollary 73. For any $u, v \in \mathcal{V}$, one has the inequality:

$$
p(u, u) \cdot p(v, v) \geq p^{2}(u, v),
$$

with equality if and only if $u=v$.

Proof. Using Proposition 70, the desired inequality may be rewritten as:

$$
\left(-\check{E}_{u} \cdot \check{E}_{u}\right) \cdot\left(-\check{E}_{v} \cdot \check{E}_{v}\right) \geq\left(-\check{E}_{u} \cdot \check{E}_{v}\right)^{2}
$$

which is simply the Cauchy-Schwartz inequality implied by the positive definiteness of the intersection form $-I$ on $\check{\Lambda}_{\mathbb{R}}$. One has equality if and only if the vectors $\check{E}_{u}$ and $\check{E}_{v}$ are proportional, which is the case if and only if $u=v$.

Let us introduce a new function, which is well-defined thanks to Corollary 73 :

Definition 74. The determinant distance $d: \mathcal{V} \times \mathcal{V} \rightarrow \mathbb{R}_{+}$is defined by:

$$
d(u, v):=-\log \frac{p(u, v)}{\sqrt{p(u, u) \cdot p(v, v)}} \text {, for all } u, v \in \mathcal{V} .
$$

Remark 75. In the paper [26], Gignac and Ruggiero defined the angular distance for any normal surface singularity, as:

$$
\rho(u, v):=-\log \frac{\left(-\check{E}_{u} \cdot \check{E}_{v}\right)^{2}}{\left(-\check{E}_{u} \cdot \check{E}_{u}\right) \cdot\left(-\check{E}_{v} \cdot \check{E}_{v}\right)}, \text { for all } u, v \in \mathcal{V} .
$$


By Proposition [70, on arborescent singularities one gets $\rho=2 d$. In the sequel [25] to the present paper, concerning arbitrary normal surface singularities, we also work with the angular distance $\rho$ as a replacement of what we call here the determinant distance.

Reformulated in terms of the determinant distance, equation (7) provides:

Proposition 76. For any three vertices $u, v, w \in \mathcal{V}$ such that $v \in[u w]$, one has:

$$
d(u, v)+d(v, w)=d(u, w) .
$$

Moreover $d$ is symmetric and $d(u, v) \geq 0$, with equality if and only if $u=v$. That is, the determinant distance $d$ is an additive distance on the tree $\Gamma$, in the sense of Definition 56.

Therefore, Proposition 57 implies that:

Corollary 77. Let $S$ be an arborescent singularity. Consider any good resolution of it. Then, for any vertices $u, v, w, l \in \mathcal{V}$, one has:

$$
d(l, u)+d(v, w) \leq \max \{d(l, v)+d(u, w), d(l, w)+d(u, v)\} .
$$

Equivalently:

$$
p(l, u) \cdot p(v, w) \geq \min \{p(l, v) \cdot p(u, w), p(l, w) \cdot p(u, v)\} .
$$

Remark 78. We could have worked instead with the function $e^{-d}$, which is a multiplicative distance function, that is, a distance with values in the cancellative abelian monoid $((0,+\infty), \cdot)$, in the sense of Bandelt and Steel [3. We prefer to work instead with a classical additive distance, in order not to complicate the understanding of the reader who is not accustomed with this more general setting, which is generalized even more by Böcker and Dress [4. Note also that, as a consequence of Proposition 70 , one has:

$$
e^{-d(u, v)}=\frac{-\left(\check{E}_{u} \cdot \check{E}_{v}\right)}{\sqrt{\left(-\left(\check{E}_{u} \cdot \check{E}_{u}\right)\right) \cdot\left(-\left(\check{E}_{v} \cdot \check{E}_{v}\right)\right)}},
$$

which is the cosine of the angle formed by the vectors $\check{E}_{u}$ and $\check{E}_{v}$ with respect to the euclidean scalar product $-I$ on $\check{\Lambda}_{\mathbb{R}}$.

Note the following consequence of Proposition [58, which refines inequality (10) from Corollary 77 ,

Proposition 79. For any $u, v, w, l \in \mathcal{V}$, one has the equivalence:

$$
p(l, v) \cdot p(u, w) \leq p(l, u) \cdot p(v, w) \Longleftrightarrow[l v] \cap[u w] \neq \emptyset
$$

with equality if and only if one has moreover $[l u] \cap[v w] \neq \emptyset$.

This proposition or, alternatively, the weaker statement of Corollary 77 will imply in turn our main results announced in the introduction (that is, Corollary 84, Theorem 85 and Theorem 119).

Example 80. Let us consider a germ of arborescent singularity $S$ which has a good resolution whose dual graph is indicated in Figure 8 . All self-intersections are equal to -2 , with the exception of $E_{f} \cdot E_{f}=-3$. The genera are arbitrary. The edge determinants at each vertex are indicated in Figure 8 near the corresponding edge. For instance, $\operatorname{det}_{a,[a b]}(\Gamma)$ is the determinant of the subtree $\Gamma_{a,[a b]}$, which is the full subtree with vertices $e, b, f$. This allows to compute the determinant product of any pair of vertices. For instance:

$$
p(a, b)=\operatorname{det}_{a,[a c]}(\Gamma) \cdot \operatorname{det}_{a,[a d]}(\Gamma) \cdot \operatorname{det}_{b,[b e]}(\Gamma) \cdot \operatorname{det}_{b,[b f]}(\Gamma)=2 \cdot 2 \cdot 2 \cdot 3=24 .
$$

The matrix of determinant products $(p(u, v))_{u, v}$ is the following one, for the ordering $a<\cdots<f$ of the vertices of $\Gamma$ :

$$
\left(\begin{array}{cccccc}
28 & 24 & 14 & 14 & 12 & 8 \\
24 & 24 & 12 & 12 & 12 & 8 \\
14 & 12 & 9 & 7 & 6 & 4 \\
14 & 12 & 7 & 9 & 6 & 4 \\
12 & 12 & 6 & 6 & 8 & 4 \\
8 & 8 & 4 & 4 & 4 & 4
\end{array}\right) .
$$

Moreover, we have $\operatorname{det}(S)=4$, as may be computed easily using Proposition 68 . 


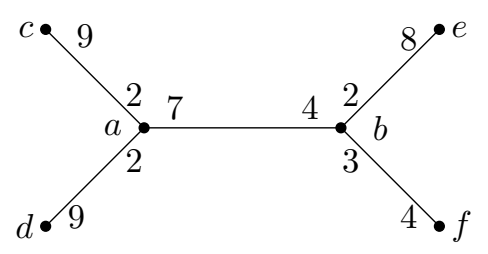

Figure 8. The edge determinants in Example 80

Remark 81. Specialize Proposition $[79$ by putting $v=w$. Then one has automatically $[l v] \cap[u w] \neq \emptyset$, which implies:

$$
p(l, v) \cdot p(u, v) \leq p(l, u) \cdot p(v, v)
$$

for any $l, u, v \in \mathcal{V}$. This inequality may be rewritten as:

$$
\left(-\check{E}_{l} \cdot \check{E}_{v}\right)\left(-\check{E}_{u} \cdot \check{E}_{v}\right) \leq\left(-\check{E}_{l} \cdot \check{E}_{u}\right)\left(-\check{E}_{v} \cdot \check{E}_{v}\right)
$$

with equality if and only if $v \in[l u]$. In [26], Gignac and Ruggiero proved that the inequality (11) is valid for all normal surface singularities, and moreover that the equality in (11) holds if and only if $v$ separates $u$ from $w$ in the dual graph. This condition is a generalization of the condition $v \in[l u]$ when $S$ is arborescent. Their result is the central ingredient of the sequel [25] of the present paper, written in collaboration with Ruggiero.

\subsection{The ultrametric associated to a branch on an arborescent singularity.}

The main results of this subsection are our generalization of Płoski's theorem to arbitrary arborescent singularities (Theorem 85) and the interpretation of the associated rooted trees as convex hulls in dual graphs of resolutions (Theorem 87).

Recall the notation $U_{L}$ explained in the introduction:

Notation 82. Let $S$ be a normal surface singularity and let $L$ be a fixed branch on it. If $A, B$ denote two branches on $S$ different from $L$, define:

$$
U_{L}(A, B):=\left\{\begin{array}{lll}
\frac{(L \cdot A)(L \cdot B)}{A \cdot B}, & \text { if } & A \neq B \\
0, & \text { if } & A=B .
\end{array}\right.
$$

The following proposition explains several ways to compute or to think about $U_{L}$ in the case of arborescent singularities (recall that the notation $u(A)$ was introduced in Definition 13):

Proposition 83. Let $S$ be an arborescent singularity and let $L$ be a fixed branch on it. Assume moreover that $A, B$ are two distinct branches on $S$ different from $L$ and that $\tilde{S}$ is an embedded resolution of $A+B+L$, with dual tree $\Gamma$. Denote $a=u(A), b=u(B), l=u(L)$. Then:

1. $U_{L}(A, B)=\operatorname{det}(S)^{-1} \cdot \frac{p(l, a) \cdot p(l, b)}{p(a, b)}$.

2. $U_{L}(A, B)=\operatorname{det}(S)^{-1} \cdot \frac{p^{2}\left(l, a \wedge_{l} b\right)}{p\left(a \wedge_{l} b, a \wedge_{l} b\right)}$.

3. $U_{L}(A, B)=\operatorname{det}(S)^{-1} \cdot p(l, l) \cdot e^{-2 h_{l}\left(a \wedge_{l} b\right)}$, where $h_{l}$ is the remoteness function on $\Gamma$ associated to the determinant distance $d$ and the root $l$, as explained in Definition [59.

Proof. Let us prove point 1. By Corollary 20, $A \cdot B=-\check{E}_{a} \cdot \check{E}_{b}$. By Proposition $70,-\check{E}_{a} \cdot \check{E}_{b}=$ $\operatorname{det}(S)^{-1} \cdot p(a, b)$. Using the analogous formulae in order to transform the intersection numbers $L \cdot A$ and $L \cdot B$, we get the desired equality.

We prove now point 2. Given the equality of the previous point, the second equality is equivalent to:

$$
p(l, a) \cdot p(l, b) \cdot p\left(a \wedge_{l} b, a \wedge_{l} b\right)=p(a, b) \cdot p^{2}\left(l, a \wedge_{l} b\right)
$$


But this may be obtained by multiplying termwise the following special cases of formula (6) stated in Proposition 69 (in which, for simplicity, we have denoted $c:=a \wedge_{l} b$ ):

$$
\begin{aligned}
& p(l, a) \cdot p(c, c)=p(l, c) \cdot p(c, a), \\
& p(l, b) \cdot p(c, c)=p(l, c) \cdot p(c, b), \\
& p(a, c) \cdot p(c, b)=p(a, b) \cdot p(c, c) .
\end{aligned}
$$

Finally, let us prove point 3. Using Definition [74, the equality (83) may be rewritten as:

$$
U_{L}(A, B)=\frac{p(l, l)}{\operatorname{det}(S)} \cdot \frac{p^{2}\left(l, a \wedge_{l} b\right)}{p(l, l) \cdot p\left(a \wedge_{l} b, a \wedge_{l} b\right)}=\frac{p(l, l)}{\operatorname{det}(S)} \cdot e^{-2 d\left(l, a \wedge_{l} b\right)} .
$$

But, by Definition [59, $d\left(l, a \wedge_{l} b\right)=h_{l}\left(a \wedge_{l} b\right)$, and the formula is proved.

The first equality stated in Proposition 83 allows to compute the maximum of $U_{L}$ :

Corollary 84. Whenever $L, A, B$ are three pairwise distinct branches on the arborescent singularity $S$, one has:

with equality if and only if $l \in[a b]$.

$$
U_{L}(A, B) \leq-\check{E}_{l} \cdot \check{E}_{l}
$$

Proof. As $[l a] \cap[l b] \neq \emptyset$, Proposition 79 implies that: $p(l, a) \cdot p(l, b) \leq p(l, l) \cdot p(a, b)$. Combining this with the first equality stated in Proposition 83 , we get:

$$
U_{L}(A, B) \leq p(l, l) \cdot \operatorname{det}(S)^{-1}=-\check{E}_{l} \cdot \check{E}_{l},
$$

where the last equality is a consequence of Proposition 70. The fact that one has equality if an only if $l \in[a b]$ follows from Proposition 79 .

Recall that $\mathcal{B}(S)$ denotes the set of branches on $S$. The following is our generalization of Płoski's theorem recalled at the beginning of the introduction:

Theorem 85. For any four pairwise distinct branches $(L, A, B, C)$ on the arborescent singularity $S$, one has:

$$
U_{L}(A, B) \leq \max \left\{U_{L}(A, C), U_{L}(B, C)\right\}
$$

Therefore, the function $U_{L}$ is an ultrametric on $\mathcal{B}(S) \backslash\{L\}$.

Proof. We will give two different proofs of this theorem.

The first proof. Let $\pi: \tilde{S} \rightarrow S$ be an embedded resolution of $A+B+C+L$. By Proposition 18. we know that the pairwise intersection numbers on $S$ of the four branches are the opposites of the intersection numbers on $\tilde{S}$ of their exceptional transforms by the morphism $\pi$. By Lemma 19, there exist four (possibly coinciding) indices $l, a, b, c \in \mathcal{V}$ such that $\left(\pi^{*} A\right)_{e x}=-\check{E}_{a},\left(\pi^{*} B\right)_{e x}=-\check{E}_{b},\left(\pi^{*} C\right)_{e x}=$ $-\check{E}_{c},\left(\pi^{*} L\right)_{e x}=-\check{E}_{l}$. Using the first equality of Proposition 83 , the stated inequality is equivalent to:

$$
\frac{p(l, a) \cdot p(l, b)}{p(a, b)} \leq \max \left\{\frac{p(l, a) \cdot p(l, c)}{p(a, c)}, \frac{p(l, b) \cdot p(l, c)}{p(b, c)}\right\} .
$$

By taking the inverses of the three fractions and multiplying then all of them by $p(l, a) \cdot p(l, b) \cdot p(l, c)$, we see that the previous inequality is equivalent to:

But this inequality is true by Corollary 77

$$
p(l, c) \cdot p(a, b) \geq \min \{p(l, b) \cdot p(a, c), p(l, a) \cdot p(b, c)\} .
$$

The second proof. We could have argued also by combining the third equality of Proposition 83 with Proposition 62. Indeed, the function $s:\left(\mathbb{R}_{+}, \leq\right) \rightarrow\left(\mathbb{R}_{+}^{*}, \leq\right)$ defined by: $s(x):=\frac{p(l, l)}{\operatorname{det}(S)} \cdot e^{-2 x}$, is strictly decreasing. This line of reasoning may be easily followed on Figure 9. Up to permuting $a, b, c$, it represents the generic tree $[l a b c]$. All other topological possibilities are degenerations of it. Using the third equality of Proposition 83, we have:

$$
\begin{aligned}
U_{L}(A, B) & =\operatorname{det}(S)^{-1} \cdot p(l, l) \cdot e^{-2 h_{l}\left(a \wedge_{l} b\right)}, \\
U_{L}(A, C)=U_{L}(B, C) & =\operatorname{det}(S)^{-1} \cdot p(l, l) \cdot e^{-2 h_{l}\left(b \wedge_{l} c\right)} .
\end{aligned}
$$




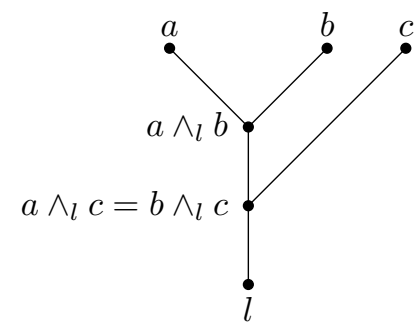

Figure 9. A generic position of $a, b, c$ and $l$.

But the inequality $b \wedge_{l} c \preceq_{l} a \wedge_{l} b$ implies that: $h_{l}\left(b \wedge_{l} c\right) \leq h_{l}\left(a \wedge_{l} b\right)$. Therefore:

$$
U_{L}(A, C)=U_{L}(B, C) \geq U_{L}(A, B),
$$

which is the ultrametric inequality (recall Proposition 42 (2)).

Remark 86. The previous theorem was proved in this form for smooth complex germs $S$ and $L$ by Chądzyński and Płoski [10, Section 4] and again by Favre and Jonsson in [20, Lemma 3.56] for smooth germs $S$ and $L$. Abío, Alberich-Carramiñana and González-Alonso later explored in [1] the values taken by this ultrametric, also in the case of smooth germs. Their results were extended recently by the first author and Płoski [23] to smooth surfaces defined over algebraically closed fields of positive characteristic. Favre and Jonsson were not conscious about the result of Chądzyński and Płoski, and they attributed the theorem to the first author's thesis [21, Cor. 1.2.3]. This last reference states in fact that a related function is an ultrametric, a result which combined with [21, Prop. 1.2.2] implies indeed that $U_{L}$ is an ultrametric. Note that at the time of writing [21, the first author did not know the papers [42] and [10].

As a consequence of Proposition 63, one gets also the announced topological interpretation of the two rooted trees associated to the ultrametric $U_{L}$ (see Definition 45):

Theorem 87. Let $S$ be an arborescent singularity and $L$ a fixed branch on it. Assume that $\mathcal{F}$ is a finite set of branches on $S$, all of them different from $L$. Consider an embedded resolution of the sum of $L$ and of the branches in $\mathcal{F}$. Let $\Gamma_{L}(\mathcal{F})$ be the dual tree of the total transform of this divisor. Then we have:

(1) the end-rooted tree $\hat{T}^{U_{L}}$ associated to the ultrametric space $\left(\mathcal{F}, U^{L}\right)$ is isomorphic as a rooted tree with leaf set $\mathcal{F}$ with the convex hull of $\{L\} \cup \mathcal{F}$ in $\Gamma_{L}(\mathcal{F})$, endowed with its topological vertex set and with root at $L$;

(2) the previous isomorphism sends the interior-rooted tree $T^{U_{L}}$ associated to the ultrametric space $\left(\mathcal{F}, U^{L}\right)$ onto the convex hull of $\mathcal{F}$ in $\Gamma_{L}(\mathcal{F})$.

Remark 88. Note that the root $L$ and the branches in $\mathcal{F}$ are always ends of $\Gamma_{L}(\mathcal{F})$. This is the reason why we have decided to associate systematically to an ultrametric a rooted tree in which the root is an end, its end-rooted tree (see Definition 45). Note also that the convex hull $[\{u(L)\} \cup\{u(A), A \in \mathcal{F}\}]$ inside $\Gamma_{L}(\mathcal{F})$, which is equal to the core of the end-rooted tree $\Gamma_{L}(\mathcal{F})$, is equipped with the additive distance $d$ of Definition 74. This fact has to be used when one deduces Theorem 87 from Proposition 63 .

Example 89. Let us consider an arborescent singularity $S$ as in Example80, That is, we assume that it admits a good resolution $\tilde{S}$ with weighted dual graph $\Gamma$ as in Figure 8 . Consider branches $L, A, B, C, D, E, F$ on $S$ such that the total transform of $L+A+\cdots+F$ on $\widetilde{S}$ is a normal crossings divisor. Moreover, we assume that the strict transforms of $A, \ldots, F$ intersect $a, \ldots, f$ respectively, and that the strict transform of $L$ intersects $E_{a}$. Therefore, with the notations of Proposition 83 , we have $l=a$. We see on Figure 8 that when $x, y$ vary among $\{a, \ldots, f\}$ and remain distinct, their infimum $x \wedge_{l} y$ relative to the root $l=a$ of $\Gamma$ is either $a$ or $b$. By the second equality of Proposition 83 , we deduce that the only possible values of $\operatorname{det}(S) \cdot U_{L}(X, Y)$, when $X \neq Y$ vary among $\{A, \ldots, F\}$, are:

$$
\frac{p^{2}(a, a)}{p(a, a)}=p(a, a)=28, \quad \frac{p^{2}(a, b)}{p(b, b)}=\frac{24^{2}}{24}=24 \text {. }
$$



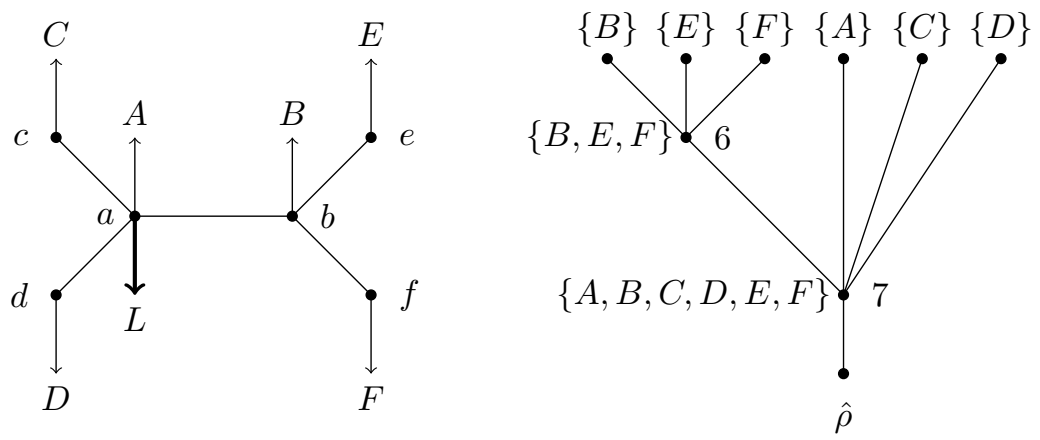

Figure 10. An illustration of Theorem 87. Example 89.

Continuing to use the second equality of Proposition 83, we get the following values for the entries of the matrix $\left(U_{L}(X, Y)\right)_{X, Y}$ (recall from Example 80 that $\left.\operatorname{det}(S)=4\right)$ :

$$
\left(\begin{array}{llllll}
0 & 7 & 7 & 7 & 7 & 7 \\
7 & 0 & 7 & 7 & 6 & 6 \\
7 & 7 & 0 & 7 & 7 & 7 \\
7 & 7 & 7 & 0 & 7 & 7 \\
7 & 6 & 7 & 7 & 0 & 6 \\
7 & 6 & 7 & 7 & 6 & 0
\end{array}\right) .
$$

One may check immediately on this matrix that $U_{L}$ is an ultrametric on the set $\{A, \ldots, F\}$. In Figure 10 we represent both the dual tree $\hat{\Gamma}$ of the total transform of $L+A+\cdots+F$ and the end-rooted tree $\hat{T}^{U_{L}}$ associated to the ultrametric $U_{L}$. Near the two nodes of $\hat{T}^{U_{L}}$ we indicate both the corresponding clusters and their diameters (as in Figure 4).

In the introduction we recalled the following result of Teissier [45, page 40], which inspired us to formulate Corollary 84

Proposition 90. If $S$ is a normal surface singularity, $A, B$ are two divisors without common branches on it and $m_{O}$ denotes the function which gives the multiplicity at $O$, then one has the inequality:

$$
\frac{m_{O}(A) \cdot m_{O}(B)}{A \cdot B} \leq m_{O}(S)
$$

This result suggests to consider the following analog of the function $U_{L}$ introduced in Notation 82

Notation 91. Let $S$ be a normal surface singularity. If $A, B$ denote two branches on $S$, define:

$$
U_{O}(A, B):=\left\{\begin{array}{lll}
\frac{m_{O}(A) \cdot m_{O}(B)}{A \cdot B}, & \text { if } & A \neq B, \\
0, & \text { if } & A=B .
\end{array}\right.
$$

An immediate consequence of the previous results is the following analog of Theorem 85 (which holds for a restricted class of arborescent singularities):

Theorem 92. For any three pairwise distinct branches $(A, B, C)$ on the arborescent singularity $S$ with irreducible generic hyperplane section, one has:

$$
U_{O}(A, B) \leq \max \left\{U_{O}(A, C), U_{O}(B, C)\right\} .
$$

Therefore, the function $U_{O}$ is an ultrametric on the set $\mathcal{B}(S)$ of branches of $S$.

Proof. By definition, a generic hyperplane section of a normal surface singularity $S$ is the divisor defined by a generic element of the maximal ideal of the local ring of $S$. For instance, if $S$ is smooth, the generic hyperplane sections are smooth branches on $S$. 
Fix an embedding of $S$ in a smooth space $\left(\mathbb{C}^{n}, 0\right)$. Choose a generic hyperplane $H$ in this space which is transversal to the three branches $A, B, C$. Therefore, its intersection numbers with the branches are equal to their multiplicities. Denote by $L$ the intersection of $S$ and $H$, which is a branch by hypothesis. Since the intersection number of a branch with $H$ in the ambient smooth space $\left(\mathbb{C}^{n}, 0\right)$ is equal to the intersection number of the branch with $L$ on $S$, we get:

$$
m_{O}(A)=L \cdot A, \quad m_{O}(B)=L \cdot B, \quad m_{O}(C)=L \cdot C .
$$

Therefore:

$$
U_{O}(A, B)=U_{L}(A, B), \quad U_{O}(A, C)=U_{L}(A, C), \quad U_{O}(B, C)=U_{L}(B, C) .
$$

We conclude using Theorem 85 .

Remark 93. Assume that $S$ is a rational surface singularity and that $\tilde{S}$ is a resolution of it. Then the exceptional transform on $\tilde{S}$ of a generic hyperplane section $L$ of $S$ is the fundamental cycle $Z_{f}$ of the resolution, defined by Artin [2, Page 132] (see also Ishii [31, Definition 7.2.3]). The total transform of $L$ is in this case a normal crossings divisor. The number of branches of $L$ whose strict transforms intersect a component $E_{u}$ of $E$ is equal to the intersection number $-Z_{f} \cdot E_{u}$. Therefore, the generic hyperplane section is irreducible if and only if all these numbers vanish, with the exception of one of them, which is equal to 1 (that is, if and only if there exists $u \in \mathcal{V}$ such that $Z_{f}=-\check{E}_{u}$ ). This may be easily checked. For instance, starting from the list of rational surface singularities of multiplicity 2 or 3 given at the end of Artin's paper 2, one sees that among the rational double points $A_{n}, D_{n}, E_{n}$, only those of type $A_{n}$ do not have irreducible generic hyperplane sections. And among rational triple points, those which do not have irreducible generic hyperplane sections are the first three of the left column and the first one of the right column of that paper.

Remark 94. Under the hypothesis of Theorem 92. Teissier's inequality stated in Proposition 90 may be proved as a particular case of the inequality stated in Corollary 84. Indeed, arguing as in the proof of Theorem 92 we may assume that we work with an irreducible hyperplane section $L$ such that the equalities (12) hold. Let $L^{\prime}$ be a second hyperplane section, whose strict transform to the resolution with which we work is disjoint from the strict transform of $L$, but intersects the same component $E_{l}$. Moreover, we may assume that both are transversal to $E_{l}$. By Corollary 20, we have $L \cdot L^{\prime}=-E_{l}^{2}$. But $L \cdot L^{\prime}=m_{O}(S)$. This shows, as announced, that our inequality becomes Teissier's one.

\subsection{Płoski's theorem and the ultrametric nature of Eggers-Wall trees.}

In this subsection we assume that both the surface $S$ and the branch $L$ are smooth. Consider a finite set $\mathcal{F}$ of branches on $S$, distinct from $L$. We explain first how to associate to them a rooted tree $\Theta_{L}(\mathcal{F})$, whose set of leaves is labeled by the elements of $\mathcal{F}$ and whose root is labeled by L: the Eggers-Wall tree of $\mathcal{F}$ relative to $L$. Then we prove that the function $U_{L}$ is an ultrametric on $\mathcal{F}$ with associated end-rooted tree isomorphic to $\Theta_{L}(\mathcal{F})$, by showing that in restriction to $\mathcal{F}$, the function $U_{L}$ corresponds to a depth function on $\Theta_{L}(\mathcal{F})$. Note that the content of this subsection cannot be extended to algebraically closed fields of positive characteristic, because in this setting there are Weiertrass polynomials whose roots are not expressible as Newton-Puiseux series (see the related Remark 114).

Choose a coordinate system $(x, y)$ on $S$ such that $L$ is defined by $x=0$.

The following considerations on characteristic exponents are classical. One may find information about their historical evolution in [24, Section 2].

Let $A$ be a branch on $S$ different from $L$. Relative to the coordinate system $(x, y)$, it may be defined by a Weierstrass polynomial $f_{A} \in \mathbb{C}[[x]][y]$, which is unitary, irreducible and of degree $d_{A}=L \cdot A$. For simplicity, we mention only the dependency on $A$, not on the coordinate system $(x, y)$.

By the Newton-Puiseux theorem, $f_{A}$ has $d_{A}$ roots inside $\mathbb{C}\left[\left[x^{1 / d_{A}}\right]\right]$ (the Newton-Puiseux roots of $A$ in the coordinate system $(x, y))$, which may be obtained from a fixed one of them $\xi\left(x^{1 / d_{A}}\right)$ by replacing $x^{1 / d_{A}}$ with the other $d_{A}$-th roots of $x$ (here $\xi \in \mathbb{C}[[t]]$ denotes a formal power series with non-negative integral exponents). Therefore, all the Newton-Puiseux roots have the same exponents. Some of those exponents may be distinguished by looking at the differences of roots: 


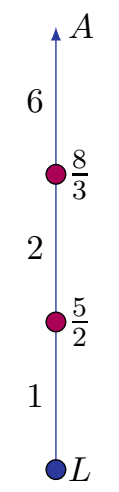

Figure 11. The Eggers-Wall tree of the series of Example 97

Definition 95. The characteristic exponents of $A$ relative to $L$ are the $x$-orders $\nu_{x}\left(\eta-\eta^{\prime}\right)$ of the differences between Newton-Puiseux roots $\eta, \eta^{\prime}$ of $A$ in the coordinate system $(x, y)$.

The characteristic exponents may be read from a given Newton-Puiseux root $\eta \in \mathbb{C}\left[\left[x^{1 / d_{A}}\right]\right]$ of $f_{A}$ by looking at the increasing sequence of exponents appearing in $\eta$ and by keeping those which cannot be written as a quotient of integers with the same smallest common denominator as the previous ones. In this sequence, one starts from the first exponent which is not an integer.

The Eggers-Wall tree of $A$ relative to $L$ is a geometrical way of encoding the sequences of characteristic exponents and of their successive common denominators:

Definition 96. The Eggers-Wall tree $\Theta_{L}(A)$ relative to $L$ is a compact oriented segment endowed with the following supplementary structures:

- an increasing homeomorphism $\mathbf{e}_{L, A}: \Theta_{L}(A) \rightarrow[0, \infty]$, the exponent function;

- marked points, which are by definition the points whose exponents are the characteristic exponents of $A$ relative to $L$, as well as the smallest end of $\Theta_{L}(A)$, labeled by $L$, and the greatest point, labeled by $A$.

- an index function $\mathbf{i}_{L, A}: \Theta_{L}(A) \rightarrow \mathbb{N}$, which associates to each point $P \in \Theta_{L}(A)$ the index of $(\mathbb{Z},+)$ in the subgroup of $(\mathbb{Q},+)$ generated by the characteristic exponents of $A$ which are strictly smaller than $\mathbf{e}_{L, A}(P)$.

The index $\mathbf{i}_{L, A}(P)$ may be also seen as the smallest common denominator of the exponents of a Newton-Puiseux root of $f_{A}$ which are strictly less than $\mathbf{e}_{L, A}(P)$.

Example 97. Assume that $A$ has as Newton-Puiseux root $x+x^{5 / 2}+x^{8 / 3}+x^{17 / 6}$. The set of characteristic exponents of $A$ relative to the branch $L$ defined by $x=0$ is $\mathcal{E}(A)=\{5 / 2,8 / 3\}$. The Eggers-Wall tree $\Theta_{L}(A)$ is drawn in Figure 11. We wrote the value of the exponent function near each vertex and of the index function near each edge on which it is constant.

We give now the definition of the Eggers-Wall tree associated to several branches. In addition to the characteristic exponents of the individual branches, we need to know the orders of coincidence of the pairs of branches:

Definition 98. If $A$ and $B$ are two distinct branches, which are also distinct from $L$, then their order of coincidence relative to $L$ is defined by:

$$
k_{L}(A, B):=\max \left\{\nu_{x}\left(\eta_{A}-\eta_{B}\right) \mid \eta_{A} \in Z\left(f_{A}\right), \eta_{B} \in Z\left(f_{B}\right)\right\} \in \mathbb{Q}_{+}^{*} .
$$

Informally speaking, the order of coincidence is the greatest rational number $k$ for which one may find Newton-Puiseux roots of the two branches coinciding up to that number ( $k$ excluded).

Notice that the order of coincidence is symmetric: $k_{L}(A, B)=k_{L}(B, A)$. 
Definition 99. Let $\mathcal{F}$ be a finite set of branches on $S$, different from $L$. The Eggers-Wall tree $\Theta_{L}(\mathcal{F})$ of $\mathcal{F}$ relative to $L$ is the rooted tree obtained as the quotient of the disjoint union of the individual Eggers-Wall trees $\Theta_{L}(A)$, for $A \in \mathcal{F}$, by the equivalence relation generated by the following natural gluing of $\Theta_{L}(A)$ with $\Theta_{L}(B)$ along the initial segments $\mathbf{e}_{L, A}^{-1}\left[0, k_{L}(A, B)\right]$ and $\mathbf{e}_{L, B}^{-1}\left[0, k_{L}(A, B)\right]$ :

$$
\mathbf{e}_{L, A}^{-1}(\alpha) \sim \mathbf{e}_{L, B}^{-1}(\alpha), \text { for all } \alpha \in\left[0, k_{L}(A, B)\right] .
$$

One endows $\Theta_{L}(\mathcal{F})$ with the exponent function $\mathbf{e}_{L}: \Theta_{L}(\mathcal{F}) \rightarrow[0, \infty]$ and the index function $\mathbf{i}_{L}: \Theta_{L}(\mathcal{F}) \rightarrow \mathbb{N}$ obtained by gluing the initial functions $\mathbf{e}_{L, A}$ and $\mathbf{i}_{L, A}$ respectively, for $A$ varying among the elements of $\mathcal{F}$.

It is an instructive exercise to prove that indeed the index functions of the branches of $\mathcal{F}$ get glued into a single index function on $\Theta_{L}(\mathcal{F})$. Note that, by construction, $k_{L}(A, B)=e_{L}\left(A \wedge_{L} B\right)$ for any pair $(A, B)$ of distinct branches of $\mathcal{F}$. Here $A \wedge_{L} B$ denotes the infimum of the leaves of $\Theta_{L}(\mathcal{F})$ labeled by $A$ and $B$, relative to the partial order on the vertices of $\Theta_{L}(\mathcal{F})$ defined by the root $L$ (see Notation 25).

Example 100. Consider a set $\mathcal{F}$ of branches in $\left(\mathbb{C}^{2}, 0\right)$, whose elements $C_{i}$ (where $\left.i \in\{1, \ldots, 5\}\right)$ have the following Newton-Puiseux roots $\eta_{i}$ :

$$
\begin{aligned}
& \eta_{1}=x^{2} \\
& \eta_{2}=x^{5 / 2}+x^{8 / 3} \\
& \eta_{3}=x^{5 / 2}+x^{11 / 4} \\
& \eta_{4}=x^{7 / 2}+x^{17 / 4} \\
& \eta_{5}=x^{7 / 2}+2 x^{17 / 4}+x^{14 / 3} .
\end{aligned}
$$

As before, we assume that $L$ is defined by $x=0$. Then $k_{L}\left(C_{1}, C_{2}\right)=k_{L}\left(C_{1}, C_{3}\right)=k_{L}\left(C_{1}, C_{4}\right)=$ $k_{L}\left(C_{1}, C_{5}\right)=2, \quad k_{L}\left(C_{2}, C_{4}\right)=k_{L}\left(C_{2}, C_{5}\right)=5 / 2, \quad k_{L}\left(C_{2}, C_{3}\right)=8 / 3$, $k_{L}\left(C_{3}, C_{4}\right)=k_{L}\left(C_{3}, C_{5}\right)=5 / 2, k_{L}\left(C_{4}, C_{5}\right)=17 / 4$ and the Eggers-Wall tree of $\mathcal{F}$ relative to $L$ is as drawn in Figure 12

EWfive.\{ps,eps,pdf $\}$ not found (or no BBox)

Figure 12. The Eggers-Wall tree of Example 100

Remark 101. Eggers [17] had constructed a tree which is nearly homeomorphic to the tree of Definition 45 (there may be a difference in the neighborhood of their roots, which the interested reader may find without difficulties). He did not consider on it the index function. Instead, he considered two types of edges which, given the exponent function, allowed to encode the same information as the index function. Another difference with Definition 99 is that Eggers assumed that the smooth branch $L$ is transversal to the tangents of all the branches of $\mathcal{F}$. What we call Eggers-Wall tree was introduced by Wall [46 in his reinterpretation of Eggers' work using computations of 0-chains and 1-chains supported on the tree.

Remark 102. Before Eggers' work, Kuo and $\mathrm{Lu} 33$, had introduced a related tree associated to a finite set $\mathcal{F}$ of branches on $\left(\mathbb{C}^{2}, 0\right)$, different from the $y$-axis. Namely, they represented by a segment each NewtonPuiseux root of the branches of $\mathcal{F}$, exactly as in Definition 96 . Then the construction proceeded exactly like for Eggers-Wall trees, with a slight variant. Namely, they used a general graphical convention for building dendrograms in genetics, using only horizontal and vertical segments. If one proceeds instead as in our Definition [99, one may prove that the Galois group of the extension of $\mathbb{C}[[x]]$ obtained by adjoining the roots of $\mathcal{F}$ acts on the resulting tree, and that its quotient by this action is the Eggers-Wall tree $\Theta_{L}(\mathcal{F})$. Moreover, the index of each point of $\Theta_{L}(\mathcal{F})$ is the cardinal of the fiber of this quotient map.

The fact that in the previous notations $\Theta_{L}(\mathcal{F}), \mathbf{e}_{L}, \mathbf{i}_{L}$ we mentioned only the dependency on $L$, and not the whole coordinate system $(x, y)$, comes from the following fact: 
Proposition 103. The Eggers-Wall tree $\Theta_{L}(\mathcal{F})$, seen as a rooted tree endowed with the exponent function $\mathbf{e}_{L}$ and the index function $\mathbf{i}_{L}$, depends only on the pair $(\mathcal{F}, L)$, where $L$ is defined by $x=0$.

Proof. - Assume first that $A$ is a branch distinct from $L$.

Choose some $p \in \mathbb{N}^{*}$. Let $\phi_{p}: \tilde{S} \rightarrow S$ be the cyclic cover of $S$ of degree $p$, ramified along $L$. Denote by $\tilde{O} \in \tilde{S}$ the preimage of $O \in S$. Consider then the (total) pullback $\phi_{p}^{*} A$. By computing in the coordinates $(x, y)$, with respect to which the morphism $\phi_{p}$ is simply given by $x=u^{p}, y=v$ for suitably chosen coordinates $(u, v)$ on $\tilde{S}$, one sees that this pullback has only smooth branches if and only if $p$ is divisible by $A \cdot L$.

Suppose that this is the case. Then again by computing in local coordinates, one sees that the set $\mathcal{E}(A)$ of characteristic exponents of $A$ with respect to $(x, y)$ is equal to the set of rationals of the form $\frac{1}{p} A_{i} \cdot \tilde{O} A_{j}$, where $\left(A_{i}, A_{j}\right)$ varies among the set of couples of distinct branches of $\phi_{p}^{*} A$ and the intersection numbers are computed at $\tilde{O}$. This shows that $\mathcal{E}(A)$ depends only on the pair $(A, L)$, and not on the coordinate system $(x, y)$ chosen such that $L$ is defined by $x=0$.

- Assume now that $A$ and $B$ are two different branches distinct from $L$. Take $p \in \mathbb{N}^{*}$ divisible by both $A \cdot L$ and $B \cdot L$. Again by computing in the coordinates $(x, y)$, we see that $k_{L}(A, B)$ is the maximal value of the rational numbers of the form $\frac{1}{p} A_{i} \cdot{ }^{\tilde{O}} B_{j}$, where $A_{i}$ varies among the branches of $\phi_{p}^{*} A$ and $B_{j}$ among those of $\phi_{p}^{*} B$. This shows that the order of coincidence of $A$ and $B$ with respect to $(x, y)$ depends also only on $L$.

By combining the two invariance properties we deduce the proposition.

Let us introduce a third function defined on the Eggers-Wall tree:

Definition 104. Let $A$ be a branch on $S$. The contact complexity $\mathbf{c}_{L}(P)$ of a point $P \in \Theta_{L}(A)$ is defined by:

$$
\mathbf{c}_{L}(P):=\left(\sum_{j=1}^{l} \frac{\alpha_{j}-\alpha_{j-1}}{\mathbf{i}_{j-1}}\right)+\frac{\mathbf{e}_{L}(P)-\alpha_{l}}{\mathbf{i}_{l}} .
$$

where $\alpha_{0}:=0, \alpha_{1}<\ldots<\alpha_{g}$ are the characteristic exponents of $A$ relative to $L$, that $\mathbf{i}_{j}:=\mathbf{i}\left(\alpha_{0}, \ldots, \alpha_{j}\right)$ is the value of the index function $\mathbf{i}_{L}$ in restriction to the half-open interval $\left(P_{j}=\mathbf{e}_{L}^{-1}\left(\alpha_{j}\right), P_{j+1}=\mathbf{e}_{L}^{-1}\left(\alpha_{j+1}\right)\right]$ and $\mathbf{e}_{L}(P) \in\left[\alpha_{l}, \alpha_{l+1}\right]$ is the value of the exponent function at the point $P$. The possibility $\alpha_{l}=\alpha_{0}=0$ is allowed.

Remark 105. The previous definition gives the same value to $\mathbf{c}_{L}(P)$ when $\mathbf{e}_{L}(P)=\alpha_{l}$, if we compute it by looking at $\alpha_{l}$ either as an element of $\left[\alpha_{l-1}, \alpha_{l}\right]$ or as an element of $\left[\alpha_{l}, \alpha_{l+1}\right]$.

Note that the right-hand side of the formula defining $\mathbf{c}_{L}(P)$ may be reinterpreted as an integral of the piecewise constant function $1 / \mathbf{i}_{L}$ along the segment $[L P]$ of $\Theta_{L}(A)$, the measure being the one determined by the exponent function:

$$
\mathbf{c}_{L}(P)=\int_{L}^{P} \frac{d \mathbf{e}_{L}}{\mathbf{i}_{L}} .
$$

This allows to express conversely $\mathbf{e}_{L}$ in terms of $\mathbf{c}_{L}(P)$ and $\mathbf{i}_{L}$ :

$$
\mathbf{e}_{L}(P)=\int_{L}^{P} \mathbf{i}_{L} d \mathbf{c}_{L} .
$$

Remark 106. Formulae (13) and (14) are inspired by the formulae (3.7) and (3.9) of Favre and Jonsson's book 20, relating thinness and skewness as functions on the valuative tree.

As the function $\mathbf{i}_{L}: \Theta_{L}(A) \rightarrow \mathbb{N}^{*}$ is increasing along the segment $\Theta_{L}(A)$, formulae (13) and (14) imply: 
Corollary 107. The contact complexity $\mathbf{c}_{L}$ is an increasing homeomorphism from $\Theta_{L}(A)$ to $[0, \infty]$. Moreover, it is piecewise affine and concave in terms of the parameter $\mathbf{e}_{L}$. Conversely, the function $\mathbf{e}_{L}$ is continuous piecewise affine and convex in terms of the parameter $\mathbf{c}_{L}$.

Let us consider now the case of a finite set $\mathcal{F}$ of branches. As an easy consequence of Definition 104, we get:

Lemma 108. The contact functions of the branches of $\mathcal{F}$ glue into a continuous strictly increasing surjection $\mathbf{c}_{L}: \Theta_{L}(\mathcal{F}) \rightarrow[0, \infty]$.

This allows us to formulate the following definition:

Definition 109. Assume that both $S$ and $L$ are smooth. Let $\mathcal{F}$ be a finite set of branches on $S$. The contact complexity $\mathbf{c}_{L}: \Theta_{L}(\mathcal{F}) \rightarrow[0, \infty]$ is the function obtained by gluing the contact complexities of the individual branches of $\mathcal{F}$.

We chose the name of this function motivated by the following theorem, which is a reformulation of a result of Max Noether's paper [40] (see also [47, Theorem 4.1.6]):

Theorem 110. Let $A$ and $B$ be two distinct branches of $\mathcal{F}$. Then, in terms of the partial order defined by the root $L$ on the set of vertices of $\Theta_{L}(\mathcal{F})$, one has:

$$
U_{L}(A, B)=\mathbf{c}_{L}\left(A \wedge_{L} B\right)^{-1} .
$$

As a consequence, we get the following strengthening of Płoski's theorem (what is stronger is the fact that the end-rooted tree associated to the ultrametric $U_{L}$ is isomorphic to the Eggers-Wall tree $\Theta_{L}(\mathcal{F})$ ):

Theorem 111. Let $L$ be a smooth branch on a smooth germ of surface $S$. Consider a finite set $\mathcal{F}$ of branches on $S$, distinct from $L$. Then $U_{L}$ is an ultrametric on $\mathcal{F}$ and the associated end-rooted tree is isomorphic to the Eggers-Wall tree $\Theta_{L}(\mathcal{F})$ of $\mathcal{F}$ relative to $L$.

Proof. By Lemma 108, $\mathbf{c}_{L}$ restricts to a height function on the rooted tree $\Theta_{L}(\mathcal{F})$. Therefore, its inverse $\mathbf{c}_{L}^{-1}$ is a depth function. By Lemma 48, we deduce that $U_{L}$ is an ultrametric. Using then Proposition 49, applied to the end-rooted tree $\Theta_{L}(\mathcal{F})$ depth-dated by $\mathbf{c}_{L}^{-1}$, we deduce that the end-rooted tree associated to the ultrametric $U_{L}$ on $\mathcal{F}$ is indeed isomorphic to $\Theta_{L}(\mathcal{F})$.

As a consequence of the previous theorem and of Theorem 87, we get:

Theorem 112. Let $L$ be a smooth branch on a smooth germ of surface $S$. Consider a finite set $\mathcal{F}$ of branches on $S$, distinct from $L$. Then the Eggers-Wall tree $\Theta_{L}(\mathcal{F})$ is isomorphic as a rooted tree with the convex hull of $\{L\} \cup \mathcal{F}$ in the dual graph of an embedded resolution of their sum, rooted at the strict transform of $L$.

Remark 113. It was the third author who proved first an isomorphism theorem of this kind in 43, Theorem 4.4.1]. There the isomorphism was proved by embedding the two trees in a common space and proving that the images coincided. In that work a slightly different notion of Eggers-Wall tree was used, coinciding topologically with Eggers' original definition from [17. Later, [43, Theorem 4.4.1] was refined by Wall [47, Sect. 9.4] (see also [47, Sect. 9.10, Rem. on Sect. 9.4]). Let us mention also that with the hypothesis of Theorem 112, the Eggers-Wall tree $\Theta_{L}(\mathcal{F})$ is combinatorially isomorphic to the dual graph of a partial embedded resolution of $L+\mathcal{F}$ (see [27, Section 3.4]).

Remark 114. When both $S$ and $L$ are smooth and $\mathcal{F}$ is a finite set of branches on $S$ different from $L$, the fact that $U_{L}$ is an ultrametric distance on $\mathcal{F}$ even when the base field has positive characteristic was proved before by the first author and Płoski in [22, Theorem 2.8]. The associated end-rooted tree provides, in a way, a generalization of the notion of characteristic exponents in positive characteristic introduced in Campillo's book (see [8, Chapter III]). As noted in the introduction, our approach also works for arborescent surface singularities $S$ defined over algebraically closed fields in positive characteristic, therefore $U_{L}$ is an ultrametric also in this generality, for any branch $L$ on $S$. 


\section{VAluative CONSIDERATiOnS}

In this section we recall first the notions of valuation and semivaluation on the local ring of $S$ and the natural partial order on the set of all semivaluations. We introduce the order valuations defined by irreducible exceptional divisors and the intersection semivaluations defined by the branches lying on $S$. The choice of a fixed branch $L$ on $S$ allows to define versions of the previous (semi)valuations which are normalized relative to $L$. We prove then that, for arborescent singularities, two such normalized (semi)valuations are in the same order relation as their representative points in the dual tree of an embedded resolution of them and of the branch $L$, seen as a tree rooted at $L$.

\subsection{Basic types of valuations and semivaluations.}

In this subsection we define the types of valuations and semivaluations considered in the sequel. We do not assume here that the normal surface singularity $S$ is arborescent.

Denote by $\mathcal{O}$ the local ring of $S$ and by $\mathfrak{m}$ its maximal ideal. Denote also:

$$
\overline{\mathbb{R}}_{+}:=\mathbb{R}_{+} \cup\{+\infty\}=[0,+\infty]
$$

endowed with the usual total order.

In full generality, a valuation or a semivaluation takes its values in an arbitrary totally ordered abelian group enriched with a symbol $+\infty$ which is greater than any element of the group. Here we will restrict to the special case where the totally ordered abelian group is $(\mathbb{R},+)$ :

Definition 115. A semivaluation on $\mathcal{O}$ is a map $\nu: \mathcal{O} \rightarrow \overline{\mathbb{R}}_{+}$such that:

- $\nu(g h)=\nu(g)+\nu(h)$ for all $g, h \in \mathcal{O}$.

- $\nu(g+h) \geq \min \{\nu(g), \nu(h)\}$ for all $g, h \in \mathcal{O}$.

- $\nu(1)=0$ and $\nu(0)=+\infty$.

If $\nu^{-1}(+\infty)=\{0\}$, then one says that $\nu$ is a valuation. Denote by $\operatorname{Val}(S)$ the set of valuations of $\mathcal{O}$ and by $\overline{\operatorname{Val}}(S)$ the set of semivaluations. There is a natural partial order $\leq_{\text {val }}$ on $\overline{\mathrm{Val}}(S)$, defined by:

$$
\nu_{1} \leq_{\text {val }} \nu_{2} \Longleftrightarrow \nu_{1}(h) \leq \nu_{2}(h), \forall h \in \mathcal{O} .
$$

In the sequel we will consider the following special types of valuations and semivaluations:

Definition 116. Let $L$ be a branch on $S$ and $\pi$ be an embedded resolution of it. As usual, $\left(E_{v}\right)_{v \in \mathcal{V}}$ denote the irreducible components of its exceptional divisor. Let $A$ be a branch different from $L$.

(1) If $v \in \mathcal{V}$, the $v$-order, denoted by $\operatorname{ord}_{v}: \mathcal{O} \rightarrow \overline{\mathbb{R}}_{+}$, is defined by:

$$
\operatorname{ord}_{v}(h):=\text { order of vanishing of } \pi^{*}(h) \text { along } E_{v} .
$$

(2) If $v \in \mathcal{V}$, the $v$-order relative to $L$, denoted by $\operatorname{ord}_{v}^{L}: \mathcal{O} \rightarrow \overline{\mathbb{R}}_{+}$, is defined by:

$$
\operatorname{ord}_{v}^{L}(h):=\frac{\operatorname{ord}_{v}(h)}{-\check{E}_{v} \cdot \check{E}_{l}} .
$$

(3) The $A$-intersection order, denoted by $\operatorname{int}_{A}: \mathcal{O} \rightarrow \overline{\mathbb{R}}_{+}$, is defined by:

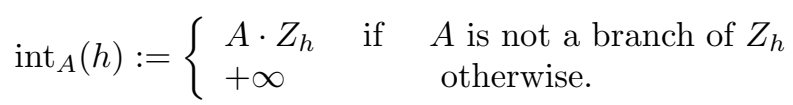

(4) The $A$-intersection order relative to $L$, denoted by $\operatorname{int}_{A}^{L}: \mathcal{O} \rightarrow \overline{\mathbb{R}}_{+}$, is defined by:

$$
\operatorname{int}_{A}^{L}(h):=\frac{\operatorname{int}_{A}(h)}{A \cdot L} .
$$

Note that the functions $\operatorname{ord}_{v}$ and $\operatorname{ord}_{v}^{L}$ are valuations, but that the functions int $A$ and int ${ }_{A}^{L}$ are semivaluations which are not valuations. Indeed, they take the value $+\infty$ on all the elements of $\mathcal{O}$ which vanish on the branch $A$. 
Remark 117. In [20], a semivaluation $\nu$ of the local ring $\mathbb{C}[[x, y]]$ is called normalized relative to the variable $x$ if $\nu(x)=1$. If $L$ denotes the branch $Z_{x}$ then, with our notations, ord ${ }^{L}$ and int ${ }_{A}^{L}$ are normalized relative to $x$. In our more general context of arbitrary normal surface singularities, the branch $L$ is not necessarily a principal divisor.

Remark 118. In [32, Sect. 7.4.8], in which $S$ is considered to be smooth, Jonsson defines a function $\alpha$ on the set of valuations of the local ring $\mathcal{O}$ which are proportional to the divisorial valuations ord a $_{u}$, by the following formula: $\alpha\left(t \cdot \operatorname{ord}_{u}\right)=t^{2}\left(\check{E}_{u} \cdot \check{E}_{u}\right)$. That is, $\alpha$ is homogeneous of degree 2 and takes the value $\check{E}_{u} \cdot \check{E}_{u}$ on the valuation $\operatorname{ord}_{u}$. In [32, Sect. 7.6.2, Note 13], he remarks that this function $\alpha$ is the opposite of the skewness function denoted with the same symbol $\alpha$ in 20. A smooth germ $S$ is arborescent and verifies $\operatorname{det}(S)=1$. Therefore, by Proposition 70 , his definition may be reexpressed in the following way in the same case of smooth germs $S$ :

$$
\alpha\left(t \cdot \operatorname{ord}_{u}\right)=-t^{2} p(u, u)=-t^{2} \operatorname{det}(S)^{-1} \cdot p(u, u) .
$$

This indicates two possible generalizations of the function $\alpha$ to arbitrary arborescent singularities, depending on which of the two previous equalities is taken as a definition.

\subsection{The valuative partial order for arborescent singularities.}

The following theorem extends Lemma 3.69 of Favre and Jonsson [20] from a smooth germ $S$ of surface and a smooth branch $L$ on it, to arborescent singularities and arbitrary branches on them:

Theorem 119. Let $L, A$ be two distinct branches on the arborescent singularity $S$. Let $\pi$ be an embedded resolution of $L+A$ and let $\Gamma_{L}(A)$ be the dual tree of the total transform of $L+A$. Consider $\Gamma_{L}(A)$ as a combinatorial tree rooted at $L$ and let $\preceq_{L}$ be the corresponding partial order. Assume that $u, v \in \mathcal{V}$. Then:

(1) $\operatorname{ord}_{u}^{L} \leq_{v a l} \operatorname{ord}_{v}^{L}$ if and only if $u \preceq_{L} v$.

(2) $\operatorname{ord}_{u}^{L} \leq_{v a l} \operatorname{int}_{A}^{L}$ if and only if $u \preceq_{L} A$.

Proof. The proof of this theorem is strongly based on the determinantal formula of Eisenbud and Neumann stated in Proposition 70 .

The proof of the implication $u \preceq_{L} v \Longrightarrow \operatorname{ord}_{u}^{L} \leq_{v a l}$ ord ${ }_{v}^{L}$ in point 1. Consider an arbitrary germ of function $h \in \mathfrak{m}$. We want to prove that $\operatorname{ord}_{u}^{L}(h) \leq \operatorname{ord}_{v}^{L}(h)$. Let us work with an embedded resolution of $L+Z_{h}$. This is no reduction of generality, as the truth of the relation $u \preceq{ }_{L} v$ does not depend on the resolution on which $E_{u}$ and $E_{v}$ appear as irreducible components of the exceptional divisor. As ord $\operatorname{ord}_{u}(h)$ is the coefficient of $E_{u}$ in the exceptional transform $\left(\pi^{*} Z_{h}\right)_{e x}$ of $Z_{h}$, the expansion (3) shows that:

$$
\operatorname{ord}_{u}(h)=\check{E}_{u} \cdot\left(\pi^{*} Z_{h}\right)_{e x} .
$$

The desired inequality $\operatorname{ord}_{u}^{L}(h) \leq \operatorname{ord}_{v}^{L}(h)$ becomes:

$$
\frac{\check{E}_{u} \cdot\left(\pi^{*} Z_{h}\right)_{e x}}{-\check{E}_{u} \cdot \check{E}_{l}} \leq \frac{\check{E}_{v} \cdot\left(\pi^{*} Z_{h}\right)_{e x}}{-\check{E}_{v} \cdot \check{E}_{l}} .
$$

The divisor $\left(\pi^{*} Z_{h}\right)_{e x}$ being a linear combination with non-negative coefficients of the divisors $\left(-\check{E}_{w}\right)_{w \in \mathcal{V}}$ (see Proposition 15), it is enough to prove that:

$$
\frac{-\check{E}_{u} \cdot \check{E}_{w}}{-\check{E}_{u} \cdot \check{E}_{l}} \leq \frac{-\check{E}_{v} \cdot \check{E}_{w}}{-\check{E}_{v} \cdot \check{E}_{l}} \text { for all } w \in \mathcal{V} .
$$

By Proposition 70, the previous inequality is equivalent to:

$$
p(l, v) \cdot p(u, w) \leq p(l, u) \cdot p(v, w) .
$$

But this last inequality is true, as a consequence of Proposition 79. Indeed, the inequality $u \preceq_{L} v$ implies that $u \in[l v] \cap[u w]$, therefore $[l v] \cap[u w] \neq \emptyset$. 
The proof of the implication $\operatorname{ord}_{u}^{L} \leq_{v a l} \operatorname{ord}_{v}^{L} \Longrightarrow u \preceq_{L} v$ in point 1. Assume by contradiction that the inequality $u \preceq_{L} v$ is not true. This means that $[l v] \cap[u u]=\emptyset$. As $[l u] \cap[v u] \neq \emptyset$ (because $u$ belongs to this intersection), Proposition 79 implies the inequality:

$$
p(l, v) \cdot p(u, u)>p(l, u) \cdot p(v, u),
$$

which may be rewritten, using Proposition [70, as:

$$
\frac{-\check{E}_{u} \cdot \check{E}_{u}}{-\check{E}_{u} \cdot \check{E}_{l}}>\frac{-\check{E}_{v} \cdot \check{E}_{u}}{-\check{E}_{v} \cdot \check{E}_{l}}
$$

Therefore, whenever the positive rational numbers $\left(\epsilon_{w}\right)_{w \in \mathcal{V} \backslash\{u\}}$ are small enough, one has also the strict inequality:

$$
\frac{-\check{E}_{u} \cdot H}{-\check{E}_{u} \cdot \check{E}_{l}}>\frac{-\check{E}_{v} \cdot H}{-\check{E}_{v} \cdot \check{E}_{l}}
$$

where $H \in \Lambda_{\mathbb{Q}}$ is defined by: $H:=\check{E}_{u}+\sum_{w \in \mathcal{V} \backslash\{u\}} \epsilon_{w} \check{E}_{w}$. As $H \in \check{\sigma}$, Proposition 16 shows that there exists $n \in \mathbb{N}^{*}$ such that $-n H$ is the exceptional transform of a principal divisor. Denote by $h \in \mathfrak{m}$ a defining function of such a divisor. Therefore, $-n H=\left(\pi^{*} Z_{h}\right)_{e x}$, and the inequality (16) implies:

$$
\frac{\check{E}_{u} \cdot\left(\pi^{*} Z_{h}\right)_{e x}}{-\check{E}_{u} \cdot \check{E}_{l}}>\frac{\check{E}_{v} \cdot\left(\pi^{*} Z_{h}\right)_{e x}}{-\check{E}_{v} \cdot \check{E}_{l}} .
$$

Using formula (15), this inequality may be rewritten as: $\operatorname{ord}_{u}^{L}(h)>\operatorname{ord}_{v}^{L}(h)$. But this contradicts the hypothesis $\operatorname{ord}_{u}^{L} \leq_{\text {val }} \operatorname{ord}_{v}^{L}$.

The proof of the implication $u \preceq_{L} A \Longrightarrow \operatorname{ord}_{u}^{L} \leq_{v a l} \operatorname{int}_{A}^{L}$ in point 2, Consider an arbitrary germ of function $h \in \mathfrak{m}$. We want to prove that $\operatorname{ord}_{u}^{L}(h) \leq \operatorname{int}_{A}^{L}(h)$. We may assume that we work with a resolution of $L+A+Z_{h}$. By Proposition [18, we have that int $A(h)=-\left(\pi^{*} A\right)_{e x} \cdot\left(\pi^{*} Z_{h}\right)_{e x}$. Using Lemma 19. we deduce the equality:

$$
\operatorname{int}_{A}(h)=\check{E}_{a} \cdot\left(\pi^{*} Z_{h}\right)_{e x},
$$

where $E_{a}$ denotes the unique component of the exceptional divisor $E$ which intersects the strict transform of $A$. By Corollary 20, $A \cdot L=-\check{E}_{a} \cdot \check{E}_{l}$. Therefore, the desired inequality $\operatorname{ord}_{u}^{L}(h) \leq \operatorname{int}_{A}^{L}(h)$ becomes:

$$
\frac{\check{E}_{u} \cdot\left(\pi^{*} Z_{h}\right)_{e x}}{-\check{E}_{u} \cdot \check{E}_{l}} \leq \frac{\check{E}_{a} \cdot\left(\pi^{*} Z_{h}\right)_{e x}}{-\check{E}_{a} \cdot \check{E}_{l}} .
$$

As before, it is enough to prove that:

$$
\frac{-\check{E}_{u} \cdot \check{E}_{w}}{-\check{E}_{u} \cdot \check{E}_{l}} \leq \frac{-\check{E}_{a} \cdot \check{E}_{w}}{-\check{E}_{a} \cdot \check{E}_{l}} \text { for all } w \in \mathcal{V} .
$$

By Proposition 70, the previous inequality is equivalent to:

$$
p(l, a) \cdot p(u, w) \leq p(l, u) \cdot p(a, w) .
$$

But this last inequality is true, as a consequence of Proposition [79, Indeed, the inequality $u \leq_{L} A$ implies that $u \in[l a] \cap[u w]$, therefore $[l v] \cap[u w] \neq \emptyset$.

The proof of the implication $\operatorname{ord}_{u}^{L} \leq_{v a l} \operatorname{int}_{A}^{L} \Longrightarrow u \preceq_{L} A$ in point 2. We reason again by contradiction, assuming that the inequality $u \preceq_{L} A$ is not true. This means that $[l a] \cap[u u]=\emptyset$. As $[l u] \cap[a u] \neq \emptyset$ (because $u$ belongs to this intersection), Proposition 79 implies that:

$$
p(l, a) \cdot p(u, u)>p(l, u) \cdot p(a, u) .
$$

Replacing $v$ by $a$ in the reasoning done in the proof of formula (17) above, we arrive at the following inequality:

$$
\frac{\check{E}_{u} \cdot\left(\pi^{*} Z_{h}\right)_{e x}}{-\check{E}_{u} \cdot \check{E}_{l}}>\frac{\check{E}_{a} \cdot\left(\pi^{*} Z_{h}\right)_{e x}}{-\check{E}_{a} \cdot \check{E}_{l}} .
$$

Combining it with formulae (15) and (18), as well as Proposition 18, it becomes: $\operatorname{ord}_{u}^{L}(h)>\operatorname{int}_{A}^{L}(h)$. But this contradicts the hypothesis $\operatorname{ord}_{u}^{L} \leq_{\text {val }} \operatorname{int}_{A}^{L}$. 
By combining Theorem 119 with Theorem 87, we get:

Corollary 120. Let $S$ be an arborescent singularity and $\mathcal{F}$ a finite set of branches on it. Let $L$ be a branch not belonging to $\mathcal{F}$. Consider any embedded resolution $\pi$ of the sum $D$ of $L$ with the elements of $\mathcal{F}$. Let $\left(E_{u}\right)_{u \in \mathcal{V}}$ be the components of the exceptional divisor of $\pi$. Then the partial order $\preceq_{v a l}$ is arborescent in restriction to the set:

$$
\left\{\operatorname{ord}_{u}^{L} \mid u \in \mathcal{V}\right\} \cup\left\{\operatorname{int}_{A}^{L} \mid A \in \mathcal{F}\right\}
$$

and the associated extended rooted tree (in the sense of Definition [28) is isomorphic with the convex hull of $\{L\} \cup \mathcal{F}$ in the dual tree of the total transform of $D$ by $\pi$, rooted at the strict transform of $L$.

Remark 121. Till now we worked with fixed embedded resolutions of the various reduced divisors considered on $S$. But, given a fixed divisor, one could consider the projective system of all its resolutions. One gets an associated direct system of embeddings of the dual graphs of total transforms. The associated ultrametric spaces are instead the same. Consider the set of all reduced divisors on $S$, directed by inclusion. One gets an associated direct system of isometric embeddings of ultrametric spaces, therefore of isometric embeddings of associated trees. One could prove then an analog of Jonsson's 32, Theorem 7.9] (which concerns only smooth germs $S$ ), which presents a valuative tree associated to the singularity $S$ (that is, a quotient of a Berkovich space) as a projective limit of dual trees. We prefer not to do this here, in order to restrict to phenomena visible on fixed resolutions of $S$ and which may be described by elementary combinatorial means, without any appeal to Berkovich geometry.

Remark 122. After having seen a previous version of this paper, Ruggiero sent us the preliminary version [26] of the paper he writes with Gignac. In that paper they extend to the spaces $\overline{\operatorname{Val}}(S)$ of semivaluations of normal surface singularities $S$, part of the theory described in [20] and [32]. This started our collaboration with Ruggiero leading to the sequel [25] of the present paper.

\section{Perspectives on non-ARborescent singularities}

In this section we give two examples, showing that for singularities which are not necessarily arborescent, $U_{L}$ is not necessarily an ultrametric or even a metric on the set of branches distinct from $L$. Then we state some open problems related with this phenomenon.

\subsection{Non-arborescent examples.}

Example 123. Consider the weighted dual graph $\Gamma$ represented in Figure 13 (the self-intersections being indicated between brackets and the genera being arbitrary). Denote by $I$ the associated intersection form. Consider the matrix of $-I$, obtained after having ordered the vertices $a, b, c, d$. By computing its principal minors, one sees that this symmetric matrix is positive definite, which shows that $I$ is negative definite. By a theorem of Grauert [28, Page 367] (see also Laufer [34, Theorem 4.9]), any divisor with normal crossings in a smooth complex surface which admits this weighted dual graph may be contracted to a normal surface singularity $S$. The graph $\Gamma$ admitting cycles, the singularity is not arborescent. Denote by $\pi$ the resolution of $S$ whose dual graph is $\Gamma$.

Let us consider branches $A, B, C, L$ on $S$ whose strict transforms by $\pi$ are smooth and intersect transversally $E_{a}, E_{b}, E_{c}, E_{l}$ at smooth points of the total exceptional divisor $E$. Therefore $\left(\pi^{*} A\right)_{e x}=-\check{E}_{a}$, $\left(\pi^{*} B\right)_{e x}=-\check{E}_{b},\left(\pi^{*} C\right)_{e x}=-\check{E}_{c}$, and $\left(\pi^{*} L\right)_{e x}=-\check{E}_{l}$.

The entries $-\left(\check{E}_{u} \cdot \check{E}_{v}\right) \cdot \operatorname{det}(S)$ of the adjoint matrix of $\left(-E_{u} \cdot E_{v}\right)_{u v}$ are as indicated in Figure 14, Using Corollary 20, one may compute then the values of $U_{L}$, getting:

$$
\begin{aligned}
& \operatorname{det}(S) \cdot U_{L}(A, B)=\frac{64 \cdot 70 \cdot 114}{6272}, \\
& \operatorname{det}(S) \cdot U_{L}(A, C)=\frac{64 \cdot 70 \cdot 114}{6440}, \\
& \operatorname{det}(S) \cdot U_{L}(B, C)=\frac{64 \cdot 70 \cdot 114}{6384} .
\end{aligned}
$$

As the three values are pairwise distinct, we see that $U_{L}$ is not an ultrametric on the set $\{A, B, C\}$. Therefore it is nor an ultrametric on the set of branches $\mathcal{B}(S) \backslash\{L\}$. Let us mention that $\operatorname{det}(S)=56$, 


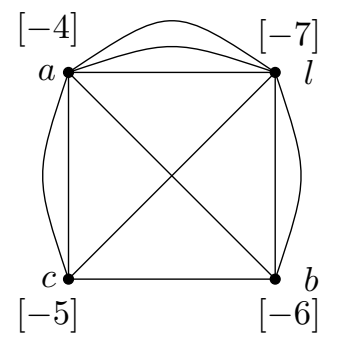

Figure 13. The dual graph of the singularity in Example 123.

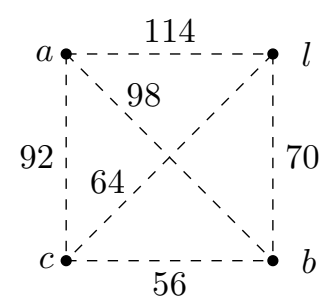

Figure 14. The label on the edge $[u v]$ is the number $-\left(\check{E}_{u} \cdot \check{E}_{v}\right) \cdot \operatorname{det}(S)$ in Example 123 ,

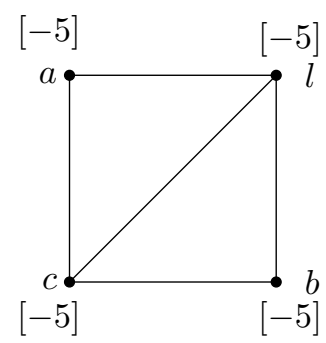

Figure 15. The dual graph of the singularity in Example 124

even if one does not need this in order to do the previous computations. One may check immediately on the above values that $U_{L}$ is nevertheless a metric on the set $\{A, B, C\}$. We do not know if it is also a metric on $\mathcal{B}(S) \backslash\{L\}$.

Example 124. Consider the weighted graph $\Gamma$ represented in Figure 15 (the self-intersections being indicated between brackets and the genera being arbitrary). As in the previous example, we see that there exist normal surface singularities with such weighted dual graphs, and that they are not arborescent.

Let us consider branches $A, B, C, L$ on $S$ with the same properties as in the previous example.

The entries $-\left(\check{E}_{u} \cdot \breve{E}_{v}\right) \cdot \operatorname{det}(S)$ of the adjoint matrix of $\left(-E_{u} \cdot E_{v}\right)_{u v}$ are as indicated in Figure 16. Using Corollary 20, one may compute then the values of $U_{L}$, getting:

$$
\operatorname{det}(S) \cdot U_{L}(A, B)=75, \quad \operatorname{det}(S) \cdot U_{L}(A, C)=35, \quad \operatorname{det}(S) \cdot U_{L}(B, C)=35 .
$$

One sees that in this case $U_{L}$ is not even a metric on the set $\{A, B, C\}$.

\subsection{Some open problems.}

Let us end this paper with some open problems:

1. Characterize the normal surface singularities for which $U_{L}$ is a metric (compare with Examples 123 and 124). 


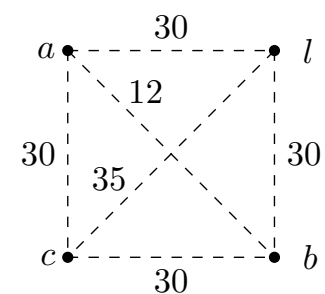

Figure 16. The label on the edge $[u v]$ is the number $-\left(\check{E}_{u} \cdot \check{E}_{v}\right) \cdot \operatorname{det}(S)$ in Example 124 .

2. Characterize the normal surface singularities whose generic hyperplane section is irreducible (compare with Theorem 92).

3. Characterize the normal surface singularities for which $U_{O}$ is an ultrametric (compare with Theorem 92).

4. Characterize the normal surface singularities for which $U_{O}$ is a metric.

Acknowledgements. This research was partially supported by the French grant ANR-12-JS01-000201 SUSI and Labex CEMPI (ANR-11-LABX-0007-01), and also by the Spanish Projects MTM2016-80659P, MTM2016-76868-C2-1-P. The third author is grateful to María Angelica Cueto, András Némethi and Dmitry Stepanov for inspiring conversations. We are also grateful to Nicholas Duchon for having sent us his thesis, and to Charles Favre, Mattias Jonsson, András Némethi, Walter Neumann and Matteo Ruggiero for their comments on a previous version of this paper.

\section{REFERENCES}

[1] Abío, I., Alberich-Carramiñana, M., González-Alonso V. The ultrametric space of plane branches. Comm. in Algebra 39 No. 11 (2011), 4206-4220.

[2] Artin, M. On isolated rational singularities of surfaces. American Journ. Math 83 (1966), 129-136.

[3] Bandelt, H.-J., Steel, M. A. Symmetric matrices representable by weighted trees over a cancellative abelian monoid. SIAM J. Discrete Math. 8 (4) (1995), 517-525.

[4] Böcker, S., Dress, S. Recovering symbolically dated, rooted trees from symbolic ultrametrics. Advances in Maths. 138 (1998), 105-125.

[5] Braun, G., Némethi, A. Surgery formula for Seiberg-Witten invariants of negative definite plumbed 3-manifolds. J. Reine Angew. Math. 638 (2010), 189-208.

[6] Buneman, P. A note on the metric properties of trees. Journ. of Combinatorial Theory 17 (1974), $48-50$.

[7] Camacho, C. Quadratic Forms and Holomorphic Foliations on Singular Surfaces. Math. Ann. 282 (1988), $177-184$.

[8] Campillo, A. Algebroid Curves in Positive Characteristic. Lecture Notes in Mathematics 813. Springer, Berlin, 1980.

[9] Caubel, C., Némethi, A., Popescu-Pampu, P. Milnor open books and Milnor fillable contact 3-manifolds. Topology 45 (2006), 673-689.

[10] Chądzyński, J., Płoski, A. An inequality for the intersection multiplicity of analytic curves. Bull. Polish Acad. Sci. Math. 36 (3-4) (1988), 113-117.

[11] Coxeter, H. S. M. Discrete groups generated by reflections. Annals of Math. 35 No. 3 (1934), 588-621.

[12] Coxeter, H. S. M. Regular polytopes. Dover Publ. Inc., New York, 1973.

[13] Dimca, A. Singularities and topology of hypersurfaces. Universitext. Springer-Verlag, New York, 1992.

[14] Duchon, N. Involutions of plumbed manifolds. Thesis, Univ. of Maryland, College Park, 1982. Available at http://sandsduchon.org/duchon/DuchonThesis.zip

[15] Du Val, P. The unloading problem for plane curves. American Journ. of Maths. 62 No. 1 (1940), 307-311.

[16] Du Val, P. On absolute and non-absolute singularities of algebraic surfaces. Revue de la Faculté des Sciences de l'Univ. d'Istanbul (A) 91 (1944), 159-215.

[17] Eggers, H. Polarinvarianten und die Topologie von Kurvensinguläritaten. Bonner Math. Schriften 147 (1983).

[18] Eisenbud, D., Neumann, W. Three-dimensional link theory and invariants of plane curve singularities. Princeton Univ. Press, 1985.

[19] Fauvet, F., Menous, F., Sauzin, D. Explicit linearization of one-dimensional germs through tree-expansions. https://hal.archives-ouvertes.fr/hal-01053805v2. Submitted on 22 Jan 2015.

[20] Favre, C., Jonsson, M. The valuative tree. Lecture Notes in Mathematics, 1853. Springer-Verlag, Berlin, 2004.

[21] García Barroso, E. Invariants des singularités de courbes planes et courbure des fibres de Milnor. PhD thesis, Univ. La Laguna, Tenerife (Spain), 1996. Available at http://ergarcia.webs.ull.es/tesis.pdf

[22] García Barroso, E., Płoski A., An approach to plane algebroid branches. Rev. Mat. Complut., 28 (1) (2015), $227-252$. 
[23] García Barroso, E., Płoski A., On the intersection multiplicity of plane branches. ArXiv:1710.05346.

[24] García Barroso, E., González Pérez, P.D., Popescu-Pampu, P. Variations on inversion theorems for Newton-Puiseux series. Math. Annalen 368 (2017), 1359-1397.

[25] García Barroso, E., González Pérez, P.D., Popescu-Pampu, P., Ruggiero, M. Ultrametric distances on valuative spaces. ArXiv:1802.01165

[26] Gignac, W., Ruggiero, M. Local dynamics of non-invertible maps near normal surface singularities. Arxiv:1704.04726

[27] González Pérez, P.D. Toric embedded resolutions of quasi-ordinary hypersurface singularities. Ann. Inst. Fourier, Grenoble 53, 6 (2003), 1819-1881.

[28] Grauert, H. Über Modifikationen und exzeptionnelle analytische Mengen. Math. Ann. 146 (1962), 331-368.

[29] Holly, J. E. Pictures of ultrametric spaces, the p-adic numbers, and valued fields. Amer. Math. Monthly 108 (2001), 721-728.

[30] Hartshorne, R. Algebraic geometry. Springer, 1977.

[31] Ishii, S. Introduction to Singularities. Springer, 2014.

[32] Jonsson, M. Dynamics on Berkovich spaces in low dimensions. In Berkovich spaces and applications, 205-366. Lecture Notes in Mathematics 2119. Springer, 2015.

[33] Kuo, T.C., Lu, Y.C. On analytic function germs of two complex variables. Topology 16 (1977), no. 4, 299-310.

[34] Laufer, H. Normal two-dimensional singularities. Annals of Mathematics Studies 71. Princeton Univ. Press, 1971.

[35] Lipman, J. Rational singularities with applications to algebraic surfaces and unique factorization. Inst. Hautes Études Sci. Publ. Math. No. 36 (1969), 195-279.

[36] Mumford, D. The topology of normal singularities of an algebraic surface and a criterion for simplicity. Inst. Hautes Études Sci. Publ. Math. No. 9 (1961), 5-22.

[37] Neumann, W. Abelian covers of quasihomogeneous surface singularities. In Singularities, Arcata 1981. Proc. Symp. Pure Math. 40 Amer. Math. Soc. 1983, 233-243.

[38] Neumann, W. On bilinear forms represented by trees. Bull. Austral. Math. Soc. 40 (1989), 303-321.

[39] Neumann, W., Wahl, J. Complete intersection singularities of splice type as universal abelian covers. Geometry and Topology 9 (2005), 699-755.

[40] Noether, M. Les combinaisons caractéristiques dans la transformation d'un point singulier. Rend.Circ.Mat.Palermo IV (1890), 89-108, 300-301.

[41] Okuma, T. Universal abelian covers of certain surface singularities. Math. Ann. 334 (2006), 753-773.

[42] Płoski, A. Remarque sur la multiplicité d'intersection des branches planes. Bulletin of the Polish Academy of Sciences. Mathematics. Volume 33 (1985), 601-605.

[43] Popescu-Pampu, P. Arbres de contact des singularités quasi-ordinaires et graphes d'adjacence pour les 3-variétés réelles. PhD thesis, Univ. Paris 7, 2001. Available at https://tel.archives-ouvertes.fr/tel-00002800v1

[44] Popescu-Pampu, P. Ultrametric spaces of branches on arborescent singularities. Math. Forsch. Oberwolfach Report 46 (2016), 2655-2658.

[45] Teissier, B. Sur une inégalité à la Minkowski pour les multiplicités. Appendix to Eisenbud, D., Levine, H. : An algebraic formula for the degree of a $C^{\infty}$-map germ. Ann. Math. 106 (1977), 19-44 (38-44).

[46] Wall, C.T.C. Chains on the Eggers tree and polar curves. Proc. of the Int. Conf. on Algebraic Geometry and Singularities (Sevilla, 2001). Rev. Mat. Iberoamericana 19 (2003), no. 2, 745-754.

[47] Wall, C.T.C. Singular points of plane curves. London Mathematical Society Student Texts, 63. Cambridge University Press, 2004.

[48] Zariski, O. The theorem of Riemann-Roch for high multiples of an effective divisor on an algebraic surface. Ann. Math. 76 No. 3 (1962), 560-615.

Departamento de Matemáticas, Estadística e I.O. Sección de Matemáticas, Universidad de La Laguna. Calle Astrofísico Francisco Sánchez, La Laguna 38200, Tenerife, España.

E-mail address: ergarcia@ull.es

Instituto de Ciencias Matemáticas (CSiC-UAM-UC3M-UCM), Departamento de Álgebra, Facultad de Ciencias Matemáticas, Universidad Complutense de Madrid, Plaza de las Ciencias 3, Madrid 28070, España.

E-mail address: pgonzalez@mat.ucm.es

Université Lille, Département de Maths., Bâtiment M2, Cité Scientifique, 59655, Villeneuve D’Ascq Cedex, FRANCE.

E-mail address: patrick.popescu@math.univ-lille1.fr 